\title{
Autophagy-based unconventional secretion of HMGB1 in glioblastoma promotes chemosensitivity to temozolomide through macrophage M1-like polarization
}

\author{
Zhuang Li ${ }^{1}$, Wen-Juan Fu' ${ }^{1}$ Xiao-Qing Chen ${ }^{1}$, Shuai Wang ${ }^{1}$, Ru-Song Deng ${ }^{1}$, Xiao-Peng Tang ${ }^{2}$, Kai-Di Yang ${ }^{1,3}$, \\ Qin Niu' ${ }^{1}$, Hong Zhou', Qing-Rui Li ${ }^{1}$, Yong Lin ${ }^{1}$, Mei Liang ${ }^{1}$, Si-Si Li ${ }^{1}$, Yi-Fang Ping ${ }^{1}$, Xin-Dong Liu', \\ Xiu-Wu Bian ${ }^{1}$ and Xiao-Hong Yao ${ }^{1 *}$
}

\begin{abstract}
Background: Glioblastoma (GB) is the most common and highly malignant brain tumor characterized by aggressive growth and resistance to alkylating chemotherapy. Autophagy induction is one of the hallmark effects of anti-GB therapies with temozolomide (TMZ). However, the non-classical form of autophagy, autophagy-based unconventional secretion, also called secretory autophagy and its role in regulating the sensitivity of GB to TMZ remains unclear. There is an urgent need to illuminate the mechanism and to develop novel therapeutic targets for GB.

Methods: Cancer genome databases and paired-GB patient samples with or without TMZ treatment were used to assess the relationship between HMGB1 mRNA levels and overall patient survival. The relationship between HMGB1 protein level and TMZ sensitivity was measured by immunohistochemistry, ELISA, Western blot and qRT-PCR. GB cells were engineered to express a chimeric autophagic flux reporter protein consisting of mCherry, GFP and LC3B. The role of secretory autophagy in tumor microenvironment (TME) was analyzed by intracranial implantation of GL261 cells. Coimmunoprecipitation (Co-IP) and Western blotting were performed to test the RAGE-NFKB-NLRP3 inflammasome pathway.

Results: The exocytosis of HMGB1 induced by TMZ in GB is dependent on the secretory autophagy. HMGB1 contributed to M1-like polarization of tumor associated macrophages (TAMs) and enhanced the sensitivity of GB cells to TMZ. Mechanistically, RAGE acted as a receptor for HMGB1 in TAMs and through RAGE-NFKB-NLRP3 inflammasome pathway, HMGB1 enhanced M1-like polarization of TAMs. Clinically, the elevated level of HMGB1 in sera may serve as a beneficial therapeutic-predictor for GB patients under TMZ treatment.
\end{abstract}

Conclusions: We demonstrated that enhanced secretory autophagy in GB facilitates M1-like polarization of TAMs to enhance TMZ sensitivity of GB cells. HMGB1 acts as a key regulator in the crosstalk between GB cells and

\footnotetext{
*Correspondence: yxh15@163.com

${ }^{1}$ Institute of Pathology and Southwest Cancer Center, Ministry of Education of China, Southwest Hospital, Third Military Medical University (Army Medical University) and Key Laboratory of Tumor Immunopathology, Chongqing, China

Full list of author information is available at the end of the article
}

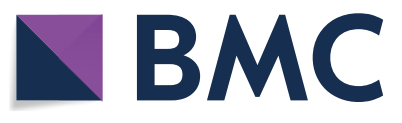

(c) The Author(s) 2022. Open Access This article is licensed under a Creative Commons Attribution 4.0 International License, which permits use, sharing, adaptation, distribution and reproduction in any medium or format, as long as you give appropriate credit to the original author(s) and the source, provide a link to the Creative Commons licence, and indicate if changes were made. The images or other third party material in this article are included in the article's Creative Commons licence, unless indicated otherwise in a credit line to the material. If material is not included in the article's Creative Commons licence and your intended use is not permitted by statutory regulation or exceeds the permitted use, you will need to obtain permission directly from the copyright holder. To view a copy of this licence, visit http://creativecommons.org/licenses/by/4.0/. The Creative Commons Public Domain Dedication waiver (http://creativeco mmons.org/publicdomain/zero/1.0/) applies to the data made available in this article, unless otherwise stated in a credit line to the data. 
tumor-suppressive M1-like TAMs in GB microenvironment and may be considered as an adjuvant for the chemotherapeutic agent TMZ.

Keywords: Glioblastoma, TMZ, Secretory autophagy, HMGB1, TAMs

\section{Background}

Glioblastoma (GB) is a heterogeneous and highly aggressive primary brain tumor with a 14.6-month patient survival $[1,2]$. The standard treatment of GB consists of maximal resection followed by radiotherapy and concomitant chemotherapy with the alkylating agent temozolomide (TMZ) [3]. The addition of TMZ improved the overall survival and progression-free survival of GB patients compared with radiotherapy alone [1]. However, the effectiveness of TMZ is limited by drug resistance. Given the high mortality and relative resistance to conventional therapy, there has been significant interest in improving the understanding of the molecular landscape and treatment of GB.

Accumulating evidence indicates that autophagy as a lysosome-mediated process plays important roles during various stages of tumorigenesis [4]. Depending on the type of cancer and the surrounding tissue context, autophagy fulfils a dual task, having either tumor-promoting or suppressing properties. Autophagy is frequently activated as a stress response by tumor cells upon TMZ treatment [5]. Autophagy, which recycles breakdown products to sustain cell metabolism and biosynthesis under stress conditions, has been proposed as a mechanism of chemoresistance to alkylating drugs [6]. Combination of autophagy inhibitors, such as bafilomycin $\mathrm{A} 1$ and chloroquine (CQ), increases chemosensitivity to TMZ in glioma cell lines. However, the effect of autophagy inhibition in combination with TMZ did not improve the overall survival of patients with GB $[5,7]$. The disputed effect of combination of autophagy inhibitors and TMZ in GB indicated that the role of autophagy in dependent on the context. Cytoprotective, cytotoxic, and cytostatic forms of autophagy induced by antitumor agents in various cancer models including GB have been reported [8-10]. The diverse roles of autophagy in cancer treatment have thus attracted considerable interest.

Recent research has revealed that certain leaderless proteins required autophagic membranes for efficient envelopment and exocytosis for unconventional secretion, named secretory autophagy $[8,9]$. The role of autophagy in protein secretion and trafficking is recognized as a function of the autophagic machinery to expand the immediate sphere of influence from intracellular compartments to extracellular environments [10-12]. One of the major breakthroughs in this area is the recognition that a subset of unconventionally secreted cytosolic proteins, such as HMGB1, IL-18, and IL-1 $\beta$, lack leader peptides and therefore cannot enter the endoplasmic reticulum-to-Golgi secretory pathway [13-15]. We have demonstrated that HMGB1 secreted by autophagic cancer-associated fibroblasts is critical for promoting the progression of luminal breast cancer [16]. HMGB1 is a highly conserved chromosomal protein and has multiple activities based on its location [17]. Inside the cells, HMGB1 sustains nucleosome dynamics and chromosomal stability and participates in DNA repair and telomere maintenance [18]. Outside the cells, HMGB1 interacts with multiple receptors to act as a cytokine and chemotactic cytokine to regulate inflammation and immunity $[19,20]$.

Extracellular HMGB1 has been reported to activate its receptors on GB cells through the downstream signaling pathway [e.g., NF-kB, IFN regulatory factor-3 (IRF3) and phosphoinositide 3-kinase (PI3K)] to produce a functional immune response, such as activation of tumor-associated dendritic cells (TADC), CD8 ${ }^{+} \mathrm{T}$ cells and macrophages [21, 22]. Besides, in the setting of bone marrow-derived macrophages, HMGB1 signaling by RAGE evoked NF $\mathrm{B}$ activation in inflammatory reaction [17]. Abundant tumor associated macrophage (TAMs) infiltration is a common feature of GBs, but these TAMs lack apparent phagocytic activity. Recent studies demonstrated that TAMs in tumor microenvironment (TME) can be categorized into M1 and M2 subtypes based on their polarization status [23, 24]. In TME, the M1 or M2 subtype TAMs represent tumorsuppressive or tumor-supportive macrophages, respectively. M1-like macrophages exert cytotoxic activity on tumor cells and elicit tumor-destructive host reactions. M2-like TAMs are generally immune-suppressive and facilitate GB malignant behavior. TAMs are crucial players in tumor-host immune interaction and cancer progression. Cytokines released by tumor cells have been proved to regulate M1 or M2-like polarization [25, 26].

However, it is unclear whether any cytokines secreted by GB cells in TME through secretory autophagy induced by TMZ affect the tumor sensitivity to chemotherapeutic agent. In this study, we report a novel finding that autophagy-based unconventional secretion of HMGB1 in GB promotes chemosensitivity to TMZ through M1-like polarization of TAMs. 


\section{Materials and methods Human GB specimens}

Human GB specimens $(n=42)$ were obtained from Southwest Hospital, Third Military Medical University (Army Medical University) in Chongqing, China, with informed consent from patients or their guardians under an approved institutional review board protocol. Histopathological diagnosis was made by at least two neuropathologists based on the World Health Organization (WHO) classification. The clinicopathological characteristics of human GB specimens were summarized in Supplementary Table 1. Paired human GB samples $(n=41)$ are from patients with primary GB and its recurrent GB under TMZ treatment which were surgically excised at Southwest Hospital, Third Military Medical University, China. The tissues and sera of GB patients were saved at Southwest Hospital Biobank (No. [2021] BC0008) and regular followup was performed for patients. This work received approval from the ethics committee of Southwest Hospital, Army Medical University in Chongqing, China (KY2020294). Frozen GB sections and formalin-fixed, paraffin-embedded GB sections were stored at $-20{ }^{\circ} \mathrm{C}$ or at room temperature, respectively. All procedures were performed in accordance with the principles of the Helsinki Declaration and approved by the institutional ethics committees.

\section{Bioinformatic analyses}

All datasets were from the following public websites: The Cancer Genome Atlas (TCGA) (https://cancergeno me.nih.gov/) and The Chinese Glioma Genome Atlas (CGGA) (http://www.cgga.org.cn/). Bioinformatic analysis plots were obtained from "GlioVis" (http://gliovis. bioinfo.cnio.es/) [27], a website for data visualization and analysis to explore gene expression data from studies involving patients with brain tumors.

GB scRNA-seq dataset was downloaded from GEO datasets (GSE84465). Raw matrix was preprocessed using computational methods deposited in the Seurat $\mathrm{R}$ package. Quality control was performed to ensure that only high-quality single-cell data was processed further, and cells with fewer than 4000 genes/cell and fewer than 8000 UMIs/cell were eliminated. Cells with greater than $20 \%$ of their transcriptome represented in mitochondrial transcripts were also excluded. We used Seurat v3 method in R v3.6 for data normalization, dimensionality reduction and clustering by default parameters. Cell types were annotated using canonical lineage makers and the expression of genes for TLR2, TLR4, TLR9, RAGE were visualized using violin polts.

\section{Cell culture}

Primary human GB1 (090116) and GB2 (20171016B) cells as well as GB cell lines U251, LN229 and mouse GB cell line GL261 were cultured in Dulbecco's Modified Eagle's Medium (DMEM) (Gibco). The primary human GB cells (GB1 and GB2) were generated in our laboratory [28-30], which isolated from two surgical specimens of GB patients (Southwest Hospital, AMU, China). The specimens were cut into $1 \times 1 \times 1 \mathrm{~mm}^{3}$ pieces and cultured in $0.2 \mathrm{ml} \mathrm{FBS}$ in a $60 \mathrm{~mm}$ dish inverted for $1 \mathrm{~h}$. The dish was then turned the right side up and supplemented with $5 \mathrm{ml}$ DMEM containing 10\% FBS. The primary GB cells were detached with $0.25 \%$ trypsin upon formation of a monolayer of cells.

Human THP-1-dreived macrophage and mouse Raw264.7 macrophage were cultured in RPMI 1640 medium (RPMI) (Gibco) and human microglia HMC3 was in MEM medium (Gibco) containing 10\% FBS (Gibco) and 1:100 penicillin-streptomycin (Hyclone) at $37^{\circ} \mathrm{C}$ in $5 \% \mathrm{CO}_{2}$.

\section{Reagents}

TMZ (No. PHR1437, Sigma) was dissolved in dimethyl sulfoxide (DMSO) at a $100 \mathrm{mM}$ stock concentration and stored at $-20^{\circ} \mathrm{C}$. Autophagy inhibitor 3-MA (No. M9281, Sigma) and LY294002 (No. S1105, Selleck Chemicals) were dissolved to $10 \mathrm{mM}$ and $100 \mu \mathrm{M}$ as stock solutions. CY-09 (No. S5774, Selleck Chemicals) and FPS-ZM1 (No. S8185, Selleck Chemicals) were at $5 \mathrm{mM}$ and $100 \mu \mathrm{M}$ as stock concentrations. Recombinant human HMGB1 (rhHMGB1, No.1690-HMB-050) was purchased from R\&D Systems and recombinant mouse HMGB1 (rmHMGB1, Cat. 50913-M01H) was from Sino Biological.

\section{qRT-PCR}

qRT-PCR was performed as previously described [16]. The specific primers for amplification were listed in Supplementary Table 2. All experiments were performed in quadruplicate samples.

\section{Co-immunoprecipitation (CO-IP) and immunoblotting}

For CO-IP, cells were lysed in Pierce IP lysis buffer (No. 87787, Thermo Fisher) and protease inhibitor cocktail (No. 04693159001, Roche). Cell lysates $(500 \mu \mathrm{l})$ were incubated with anti-HMGB1 antibody (ab18256, 1:200, Abcam) or control IgG (40 $\mu$ l Protein A/G PLUS-Agarose, No. $17061801, \mathrm{GE}$ health) overnight at $4{ }^{\circ} \mathrm{C}$. The beads were washed three times with PBS, followed by immunoblotting. Immunoblotting was performed as previously described [31]. Primary antibodies used were anti-HMGB1 (ab18256, 1:1000, Abcam), anti-SQSTM1/ p62 (88588S, 1:1000, CST), anti-ATG5 (12994 T, 
1:1000, CST), anti-ATG7 (8558 T, 1:1000, CST), antiLC3B (sc-376404, 1:1000, Santa), anti-GAPDH (5174S, 1:1000, CST), anti-RAGE (ab3611, 1:1000, Abcam), anti-GORASP2 (10598-1-AP, 1:1000, Proteintech), anti-p-ERK1/2 (8544S, 1:1000, CST), anti-IKB (9246S, 1:1000, CST), anti-NLRP3 (19771-1-AP, 1:1000, Proteintech), anti-ASC (sc-514414, 1:1000, Santa) and antip-NF-kB p65 (3033S, 1:1000, CST), anti- $\beta$-TUBULIN (2128S,1:1000, CST).

\section{Immunohistochemistry (IHC)}

Tissue slices were deparaffinized and hydrated by a series of xylene and alcohol treatment. The slices were incubated with anti-HMGB1 (ab18256, 1:200, Abcam), antiLC3B (3868S, 1:200, CST), anti-SQSTM1/p62 (88588S, 1:200, CST) and anti-Syntaxin 17 (STX17, 17815-1-AP, $1: 100$, Proteintech) at $4{ }^{\circ} \mathrm{C}$ overnight, followed by incubation with avidin-biotin-peroxidase. HMGB1 was considered positive by nucleus staining and the expression levels were semi-quantified by a composite score system based on both the percentage and intensity of stained tumor cells. The percentage of positive cells was calculated in high-power fields (HPF) as follows: $0(<10 \%$ positive tumor cells), 1 (11\%-50\% positive tumor cells), $2(51 \%-75 \%$ positive tumor cells) and 3 ( $>75 \%$ positive tumor cells). The staining was scored by two independent neuropathologists as the proportion of positive tumor cells $\times$ the staining intensity. Images were captured using a DP72 digital camera (Olympus) connected with a BX51 microscope (Olympus).

For calculating integrated optic density (IOD) values of LC3B, SQSTM1, STX17 and HMGB1 in tumor cells, five representative fields (magnification $\times 200$ ) in the region of tumor cells were randomly selected. Image-Pro Plus 6.0 software (MEDIA Cybernetics, Rockville, MD, USA) was utilized to measure the areas of tumor cell region excluding stroma areas and the IOD of the tumor cells expressing LC3B, SQSTM1, STX17 and HMGB1. Recognition of tumor cell regions was conducted under the guidance and confirmation of pathologists.

\section{Immunofluorescence (IF)}

Mouse GB xenografts were collected from mice when neurological signs occurred after GB cell transplantation. Human GB specimens were obtained from patients through surgical resection. Cultured cells or tumor sections were fixed in $4 \%$ paraformaldehyde for $15 \mathrm{~min}$ and washed with PBS twice. Samples were blocked with PBS containing $1 \%$ BSA plus $0.3 \%$ Triton X-100 for 30 min at room temperature, then incubated with indicated primary antibodies overnight at $4{ }^{\circ} \mathrm{C}$ followed by the fluorescent second antibody at room temperature for $1 \mathrm{~h}$. Nuclei were counterstained with DAPI for $5 \mathrm{~min}$, and then sections were mounted on glass and subjected to microscopy. Primary antibodies listed as follows: antiCD206 (sc-58986, 1:200, Santa), anti-CD206 (MCA2235, 1:200, Bio-Rad), anti-IBA1 (ab5076, 1: 100, Abcam), antiIBA1 (019-19741, 1:400, Wako), anti-CD16/32 (553142, 1: 200, BD Biosciences), anti-LC3B (3868S, 1: 200, CST), anti-TLR2 (JM22-41, 1:200, Thermo Fisher), anti-TLR4 (ab22048, 1: 100, Abcam), anti-TLR9 (IMG-305A, 1: 100, NOVUS), anti-RAGE (sc-365154, 1:100, Santa), anti-HMGB1 (ab18256, 1:100, Abcam), anti-HMGB1 (sc-56698, 1:100, Santa), anti-TNF- $\alpha$ (MAB610, 1: 200, NOVUS), anti-IFN- $\gamma$ (8455S, 1: 200, CST), anti-IL-1 $\beta$ (12703S, 1: 200, CST), anti-IL-6 (ab9324, 1: 100, Abcam), anti-IL-8 (MAB208, 1: 200, NOVUS), anti-CCL2 (39091S, 1: 200, CST), anti-NLRP3 (19771-1-AP, 1:100, Proteintech) and anti-ASC (sc-514414, 1:200, Santa).

\section{Transmission electron microscopy (TEM)}

GB cells were harvested after drug treatments, washed twice with PBS, and fixed with ice-cold-glutaraldehyde (3\% in $0.1 \mathrm{M}$ cacodylate buffer, $\mathrm{pH} 7.4$ ) overnight. Thin Sects. $(75 \mathrm{~nm}$ ) were prepared, stained with methylene and observed under light microscopy. All ultra-thin sections were stained with lead citrate for observation under TEM with a Hitachi HT7700 electron microscopy (Tokyo, Japan) at $80 \mathrm{kV}$.

\section{Assay for autophagy}

GB cells in $15 \mathrm{~mm}$ confocal dish plates were infected with AAV-mCherryGFP-LC3B $\quad(\mathrm{MOI}=30, \quad$ HB-AP2100001, Hanbio) according to the manufacturer's instruction. After $24 \mathrm{~h}$, cells were treated with agents as required. Six fluorescence fields were captured with confocal microscopy (LSM900, ZEISS). Green and red fluorescence represented phagophore and autolysosome, respectively and yellow puncta represented autophagosome. The autophagic activity indicated by formation of autophagosome was determined by quantifying the yellow puncta. Six fields per sample and three replicates per treatment were included.

\section{Gene silencing with siRNA and lentiviral transduction}

GB cells were seeded at $5 \times 10^{4}$ cells/well in 6-well plates. For gene knockdown, GB cells were transfected with Lipofectamine RNAiMAX (No. 13778150, Invitrogen). siRNA and Lipofectamine RNAiMAX reagent were diluted into Opti-MEM medium and mixed at a 1:1 ratio, which were incubated for $5 \mathrm{~min}$ at room temperature before adding to the cells. For ATG5 and GORASP2 depletion, cells were analyzed for $48 \mathrm{~h}$ after specific siRNA transfection. siRNA for human ATG5 (Signal Silence ${ }^{\circledR}$ Atg5 siRNA I \#6345) and control (Signal Silence ${ }^{\circledR}$ Control siRNA \#6568) was purchased from Cell 
Signaling Technology. siRNA for human GORASP2 was purchased from RiboBio Technology. Human HMGB1/ mouse Hmgb1-specific shRNA and shCtrl vectors were purchased from Hanbio Technology Corp. Ltd., China. Lentivirus packaging and transduction were conducted as described previously [31]. shRNA sequences are listed in Supplementary Table 3.

\section{ELISA}

Concentrations of HMGB1 in the culture supernatant of GB cells were determined using a Human ELISA Kit (\#ARG81185, Arigo) according to the manufacturer's instructions. IL-33, IL- $1 \alpha$, IL-37, IL-18, FGF1, FGF2, Galectin-3, MIF, Annexin A1, S100A8, IL-6 and CCL2 were measured by ELISA Kit (\#EK0929, \#EK0389, \#ЕК1363, \#ЕК0864, \#ЕК0339, \#ЕК0342, \#ЕК0764, \#ЕK0813, \#ЕK1745, \#ЕK1558, \#ЕK0410, \#ЕК0441, BOSTER). HMGB1 in GB patient sera was measured using an ELISA Kit (\#6010, Chondrex). TNF- $\alpha$, IL-8 and IFN- $\gamma$ (\#EL10019, \#EL10008 and \#EL10024, Anogen) and IL-1 $\beta$ (\#DLB50, R\&D) were measured by ELISA.

\section{Co-culture}

THP1 cell line-derived macrophages $\left(1 \times 10^{5}\right)$ and GB cell line LN229 $\left(1 \times 10^{5}\right)$ were cultured in the upper and lower chamber of Transwell Permeable Support systems (No. 353493 Corning) with a $0.4 \mu \mathrm{m}$ pore-size filter. All co-culture was conducted in the presence of TMZ $(1000 \mu \mathrm{M})$ for $24 \mathrm{~h}$.

\section{Cell viability}

For CCK-8 assay, cells were inoculated in 96-well plates and maintained in an incubator for time period. CCK-8 solution (10 $\mu \mathrm{L}, \mathrm{B} 34304$, Bimake) was added to each well for $1.5 \mathrm{~h}$ prior to measurement at OD $450 \mathrm{~nm}$.

\section{Orthotopic xenograft}

GL261 cells $\left(5 \times 10^{4}\right)$ with pLVX-puro-linker-luciferase lentivirus were injected intracranially into the right frontal lobes of 4-6-week-old male C57BL/6 mice (Laboratory Animal Center, Southwest Hospital, Third Military Medical University (Army Medical University), China). Mice were grouped by 10 animals in large plastic cages and were maintained under pathogen-free conditions. Growing xenograft tumors were detected and quantified by bioluminescence imaging using an In Vivo Image System (IVIS) (PerkinElmer, USA). Mice with neurological signs or moribund were sacrificed and tumors were collected. The animal experiments were approved by the Institutional Animal Care and Use Committee of Southwest Hospital, Third Military Medical University (Army Medical University) in accordance with the Guide for the Care and Use of Laboratory Animals.

\section{Tissue dissociation and flow cytometry analysis}

GL261 cell-derived xenograft mice with different treatment were perfused transcardially with cold phosphatebuffered saline (PBS) to clear away blood cells from the brain. The tumor-bearing brain hemispheres were dissociated enzymatically to obtain a single-cell suspension with a Brain Tumor Dissociation Kit (No. 130-095-942, Miltenyi Biotec) according to the manufacturer's protocol. The cell suspension was filtered through a $70 \mu \mathrm{m}$ strainer and centrifuged at $300 \times \mathrm{g}, 4{ }^{\circ} \mathrm{C}$ for $10 \mathrm{~min}$. Next, myelin was removed by centrifugation on $30 \%$ Percoll gradient (No. 17089101, GE Healthcare). Cells suspension was centrifuged at $1050 \times \mathrm{g}, 4^{\circ} \mathrm{C}$ for 30 min without acceleration and brakes. Finally, cells were collected for flow cytometric analysis. Cells were incubated for 15 min with True-Stain Monocyte Blocker ${ }^{\mathrm{TM}}$ (No. 426102, Biolegend) in FACS Buffer to block FcyRIII/II and reduce unspecific antibody binding. For surface marker analysis, cells were re-suspended in FACS buffer and stained with PerCP-Cyanine5.5 anti-CD45 (103132, 1:100, Biolegend), APC anti-CD11b (101212, 1:100, Biolegend), PE/Cyanine7 anti-CD86 (105013, 1:100, Biolegend), and $\mathrm{PE}$ anti-CD11c (117308, 1:100, Biolegend) at $4{ }^{\circ} \mathrm{C}$ for $30 \mathrm{~min}$. Cells were fixed and permeabilized (No. 554714, $\mathrm{BD})$ for intracellular protein staining, then labeled with FITC anti-CD206 (141704, 1:100, Biolegend). Data were acquired by the BD LSRFortessa and analyzed with FlowJo software v10.

\section{Statistical analysis}

Similar results were obtained from three independent experiments. Statistical differences were determined by two tailed unpaired Student's t-test for two groups or by two-way ANOVA for multiple groups. Results presented in this study are as the mean \pm SD. For KaplanMeier survival curves and statistical differences were determined by log-rank test. All analyses were carried out using Microsoft excel 2019 or GraphPad Prism 8.3 software. $P<0.05$ was considered statistically significant. Detailed information is described in Figure legends. Significant statistical differences were defined as ", $P<0.05$; **, $P<0.01$; ***;,$P<0.001$.

\section{Results \\ Exocytosis of HMGB1 induced by TMZ is dependent on the formation of autophagic vacuoles}

To determine whether TMZ promoted the exocytosis of leaderless proteins, the secretory autophagy proteins HMGB1, IL-33, Galectin-3, IL-1 $\alpha$, MIF, FGF1, AnnxinA1, S100A8, IL-1 $\beta$, IL-37, IL-18 and FGF2, in supernatants of primary GB cells (GB1 and GB2 cells) and cell lines (U251 and LN229) under TMZ were analyzed. HMGB1 is significantly elevated in supernatants with 
TMZ than in control without TMZ (Fig. S1). The mRNA of autophagy-related genes ATG5 (autophagy-related 5), ATG7 (autophagy-related 7), LC3B (microtubule associated protein 1 light chain 3 beta) and $B E C N 1$ were elevated in GB cells under TMZ treatment (Fig. S2A).

Primary GB1 and U251 cells were engineered to express an autophagic flux reporter protein consisting of mCherry, GFP and LC3B. As expected, TMZ enhanced the autophagic activity indicated by yellow autophagosome puncta (Fig. S2B-C) and the formation of autophagic vacuoles (Fig. S2D) in these cells.

To further detect the autophagy activity of GB cells under TMZ, we used Western blot to observe the level of LC3B and SQSTM1. LC3B, which is required for the elongation of autophagosomes and widely used as a biomarker of autophagy, has two forms: type I is cytosolic and type II is cleaved, lipidated and membrane-bound. During autophagy activation, LC3B-II increases due to the conversion of LC3B-I. The ratio of LC3B-II to LC3B$\mathrm{I}$ is considered the most reliable marker for quantification of the level of autophagy in cells. SQSTM1, another autophagy-related protein, is incorporated into the completed autophagosome and is degraded in autolysosomes, thus serving as an index of autophagic degradation. The decreased SQSTM1 levels are associated with autophagy activation [32]. When autophagy activity was elevated (the increase of LC3B-I to LC3B-II conversion and the decrease of SQSTM1) by TMZ treatment, the intracellular HMGB1 in GB cells decreased and the extracellular HMGB1 increased (Fig. 1A-B and Fig. S2E). HMGB1 was also visualized to translocate time-dependently from the nuclei to the cytoplasm and extracellular space with TMZ treatment as measured by immunofluorescence confocal microscopy (Fig. 1C, left panel). Pearson' colocalization analysis showed that HMGB1 and LC3B were colocalized (Fig. 1C, middle and right panel). Addition of autophagy inhibitors, 3-methyladenine (3-MA) and LY294002 reduced LC3B-I to LC3B-II conversion induced by TMZ associated with significant decrease in extracellular HMGB1, which was retained in intracellular regions (Fig. 1D and Fig S3A).

We additionally knocked down ATG5, a core molecular machinery component involved in autophagosome formation [33], to test the effect on secretory autophagy by GB cells (Fig. S3B). Knockdown of ATG5 in GB cells (siATG5) resulted in a marked decrease in LC3B-I to LC3B-II conversion and significant reduction of extracellular HMGB1 upon TMZ treatment (Fig. 1E and Fig. S3C). Previous studies have demonstrated that the golgi reassembly stacking protein 2 (GORASP2) facilitates autophagosome-lysosome fusion and controls the secretory autophagy $[34,35]$. We thus transiently transduced GB cells with adenovirus harboring siRNA against GORASP2 (siGORASP2-I and II) and a scrambled siRNA sequence (siCtrl) as a control. The autophagic flux (autophagosomes and autolysosomes) decreased in siGORASP2-I and II GB cells transfected with AAV-mCherryGFP-LC3B upon TMZ treatment (Fig. 1F). Western blot showed that knockdown of GORASP2 in GB cells reduced LC3B-I to LC3B-II conversion and extracellular levels of HMGB1 promoted by TMZ (Fig. 1G and Fig. S3D). These data suggest that the exocytosis of HMGB1 induced by TMZ in GB cells is dependent on the formation of autophagic vacuoles.

\section{Extracellular HMGB1 serves as a TMZ therapeutic predictor for GB patients}

To investigate the function of HMGB1 in GB patients, we analyzed mRNA levels of HMGB1 in GB and non-tumor tissues from the TCGA-GBM and Rembrandt dataset. Compared to non-tumor and low-grade glioma, mRNA levels of HMGB1 were significantly increased in GB (Fig. 2A and Fig. S4A-B). GB with high HMGB1 expression in intracellular region (including the nucleus and cytoplasm) predicted a worse overall survival rate (OS) of patient than those with lower HMGB1 expression in the tumors (Fig. 2B). The results are consistent with the

\footnotetext{
(See figure on next page.)

Fig. 1 Release of HMGB1 induced by TMZ in GB is dependent on the autophagic vacuoles. A Immunoblot of HMGB1, SQSTM1 and LC3B-I to LC3B-II conversion in primary GB1 cells (GB1 cells) treated with $1000 \mu \mathrm{MTMZ}$ (left panel). ELISA determining the HMGB1 in supernatants of primary GB1 cells (GB1 cells) (right panel). $n=3$. B Immunoblot of HMGB1, SQSTM1 and LC3B-I to LC3B-II conversion in GB1 cells treated with TMZ for $72 \mathrm{~h}$ (left panel). ELISA determining the HMGB1 in supernatants of GB1 cells (right panel). $n=3$. C Colocalization of LC3B (red) with HMGB1 (green) in GB1 cells under TMZ (left panel). Colocalization tracer profile along the line (white arrows) is indicated as merged images (middle panel). Pearson's colocalization coefficient for LC3B and HMGB1 derived from three independent experiments with five fields (right panel). Scale bars $=10 \mu \mathrm{m}$. $\mathbf{D}$ Immunoblot of HMGB1 and LC3B-I to LC3B-II conversion in GB1 cells with or without 3-MA (5 mM) and LY294002 (100 nM) for 4 h, then stimulated

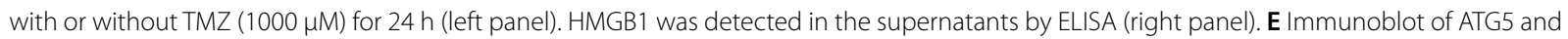
LC3B-I to LC3B-II conversion in GB1 cells transiently transfected with scrambled siRNA (siCtr) or siRNA ATG5 (siATG5) for $48 \mathrm{~h}$ then treated with TMZ $(1000 \mu \mathrm{M})$ for $24 \mathrm{~h}$ (left panel). HMGB1 in the supernatants was detected by ELISA (right panel). F GB1 cells transfected with scrambled siRNA (siCtrl) or siRNA GORASP2 (siGORASP2) for $36 \mathrm{~h}$ were transfected with AAV-mCherryGFP-LC3B then treated with TMZ (1000 $\mu \mathrm{M}$ ) for $24 \mathrm{~h}$ (left panel). The number of autophagosomes was analyzed in ten random fields for each independent experiment (right panel). G Immunoblot of GORASP2 and LC3B-I to LC3B-II conversion in GB1 cells transiently transfected with siCtrl and siGORASP2 for $48 \mathrm{~h}$ then with TMZ (1000 $\mu \mathrm{M})$ treatment for $24 \mathrm{~h}$ (left panel). HMGB1 in the supernatants was detected by ELISA (right panel). ${ }^{*} P<0.05,{ }^{* *} P<0.01$, ${ }^{* *} P<0.001, n s=$ no significance
} 


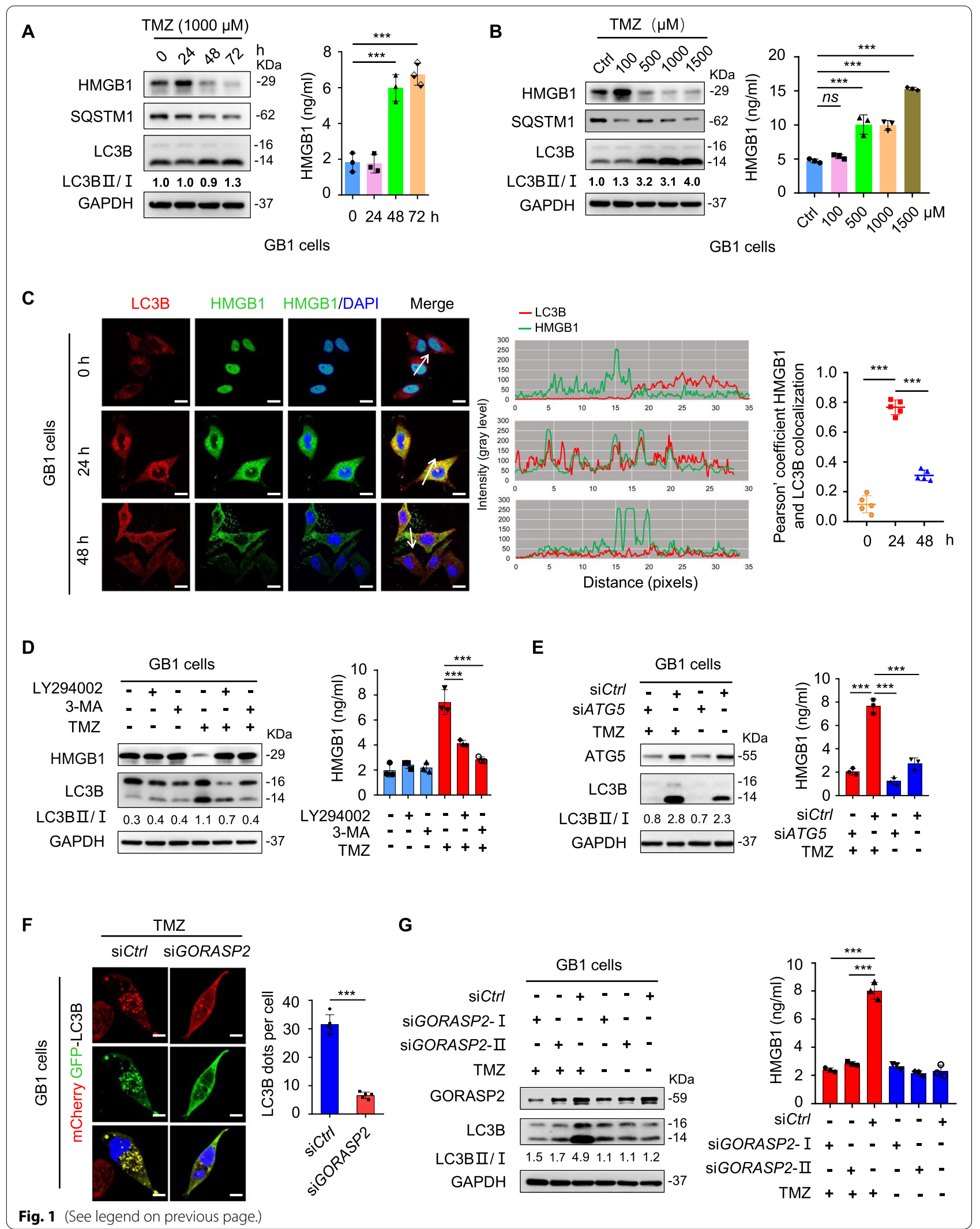


contribution of intracellular HMGB1 to DNA repair and tumorigenesis [36, 37].

To study the role of extracellular HMGB1 in GB, we collected tissues and sera of 41 patients with paired GBs. IHC staining showed that HMGB1 was mainly located in the nuclei of GB tissues without TMZ treatment, and in paired GBs with TMZ treatment, HMGB1 was positioned in the extracellular region (Fig. 2C, left panel). Quantitative analysis showed that the IHC scores of intracellular HMGB1 were significantly reduced in GB tissues upon TMZ treatment $(n=41)$ (Fig. $2 \mathrm{C}$, right panel). We further measured the HMGB1 in sera of paired GB patients. Compared with GBs without TMZ, the levels of HMGB1 in sera significantly enhanced in GBs with TMZ treatment (Fig. 2D). Therefore, TMZ cause the translocation of HMGB1 from intracellular to extracellular regions in GB. Kaplan-Meier analysis demonstrated that GB patients who received TMZ treatment with high HMGB1 in sera displayed a favorable OS than those with lower HMGB1 in sera (Fig. 2E). Therefore, our studies indicate that HMGB1 in the intracellular region of GB is detrimental to the survival of patients. However, when HMGB1 is released into the extracellular space upon TMZ, it might be beneficial for GB patients. Thus, HMGB1 based on its location may function as a prognostic and treatment-predictor for GB patients.

To determine the correlation between autophagy and HMGB1 in GB tissues, we observed the expression of autophagy proteins and the location of HMGB1 in human GB specimens (GB7225, GB1388) with TMZ treatment. In GB7225 specimen with high level of autophagy (higher expression of LC3B, STX17 and lower expression of SQSTM1), HMGB1 in the nuclei and cytoplasm of tumor cells was significantly diminished. However, in GB1388 specimen with lower levels of autophagy (lower expression of LC3B, STX17 and higher expression of SQSTM1), HMGB1 accumulated in the nuclei and cytoplasm of tumor cells (Fig. 2F-G). These results suggest that extracellular HMGB1-dependent secretory autophagy prolonged the overall survival of GB patients, which may elevate the sensitivity of TMZ. This is consistent with recent reports showing that extracellular HMGB1 acting as a cytokine or chemotactic cytokine stimulates antitumor immunity responses during chemotherapy or radiotherapy [38-40].

\section{HMGB1 contributes to M1-like polarization of macrophages in GB}

HMGB1 interacts with receptors to act as a cytokine or chemokine to regulate inflammation and immunity. Reported receptors for HMGB1 included TLR2, TLR4, TLR9 and RAGE [17, 38-40]. We analyzed the expression of these putative HMGB1 receptorsin human GB specimens by single cell sequencing and found that these molecules mainly located in tumor associated macrophages (TAMs) in the microenvironment (Fig. 3A).

Previous studies have demonstrated that tumor microenvironment (TME) of GBs contains abundant TAMs, including both tumor-supportive macrophages (M2-like TAMs) and tumor-suppressive macrophages (M1-like TAMs) [41]. Immunofluorescence showed that TAMs marked by IBA1 in TME of human GB samples (GB6429) without TMZ treatment expressed HMGB1 receptors TLR2, TLR4, TLR9 and RAGE (Fig. 3B). We then cultured human THP1 cell line-derived macrophages, mouse RAW264.7 macrophages and human microglia HMC3 with rhHMGB1 or rmHMGB1. The mRNA levels of macrophage subpopulation markers, including these specificity for M1-like macrophages (IFN- $\gamma, T N F-\alpha$, iNOS, IL-1 $\beta, C D 68, C c l 2, I l-6$, Ptgs2) and M2-like macrophages (CCL22, CSF2, VEGF, CD163, CD206, ARG1, Ccl24, Ccr2, Cd200r1, Chil3, Mrc1, Pparg) were analyzed by qRT-PCR. In THP1 cell line-derived and RAW264.7 macrophages cultured with rhHMGB1 or rmHMGB1, M1-like related mRNA was significant upregulated but M2-like related mRNA was downregulated (Fig. 3C-D). However, in HMC3 cells, M1-like and M2-like mRNA were not changed (Fig. S5).

To explore whether HMGB1 secreted by GB cells upon TMZ treatment exerted function on TAMs through paracrine mechanisms, we knocked down HMGB1 with short hairpin RNA (shRNA) in a GB cell line LN229 and GL261 (Fig. S6). Co-culture of THP1 cell line-derived macrophages with shHMGB1-LN229 cells with or

\footnotetext{
(See figure on next page.)

Fig. 2 The correlation between HMGB1 levels and the prognosis of GB patients treated with TMZ. A The mRNA levels of HMGB1 in non-tumor tissues $(n=14)$ and GB tissues $(n=528)$ from TCGA-GBM/HGU133 datasets (left panel), as well as in WHO II $(n=226)$, WHO III $(n=244)$ and WHO IV $(n=150)$ grade glioma tissues from TCGA-LGG/GBM datasets (right panel). B The overall survival (OS) rates of mRNA HMGB $1^{\text {low }}$ and HMGB $1^{\text {high }}$ GB patients from CGGA datasets $(n=489)$. C Representative immunohistochemistry (IHC) of HMGB1 in paired GB patients (left panel) with quantification (right panel). HMGB1 stains brown color. $n=41$, Scale bars $=50 \mu \mathrm{m}$. D The concentration of HMGB1 in sera of paired GB patients. $n=41$. $\mathbf{E}$ The OS rate of TMZ-treated serum HMGB1 $1^{\text {low }}$ and $H M G B 1^{\text {high }}$ GB patients. $n=41$. F Representative $I H C$ of LC3B, SQSTM1, STX 17 and HMGB1 in serial sections of GB specimens (GB7225 and GB1338) with TMZ treatment. Areas examined under higher magnification were marked. Brown color is positive. Scale bars $=50 \mu \mathrm{m}$. G The correlation between LC3B and HMGB1 expression in GB patients with TMZ treatment ( $n=41)$ (upper panel). The correlation between SQSTM1 and HMGB1 expression in GB patients with TMZ treatment $(n=41)$ (lower panel). Pearson's correlation test. ${ }^{*} P<0.05,{ }^{* *} P<0.01,{ }^{* * *} P<0.001, n s=$ no significance
} 
A

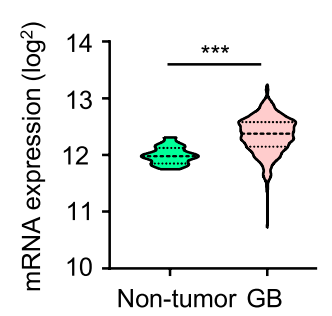

TCGA-GBM/HGU-133

Non-tumor $(n=14)$ GB $(n=528)$

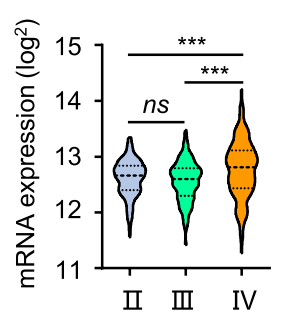

$(n=226)(n=244)(n=150)$

TCGA-LGG/GBM
B

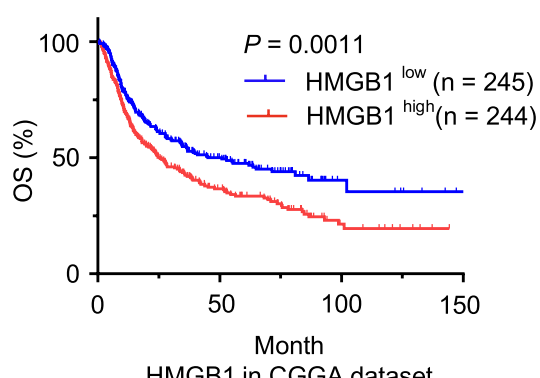

Case 5

Case 6

C

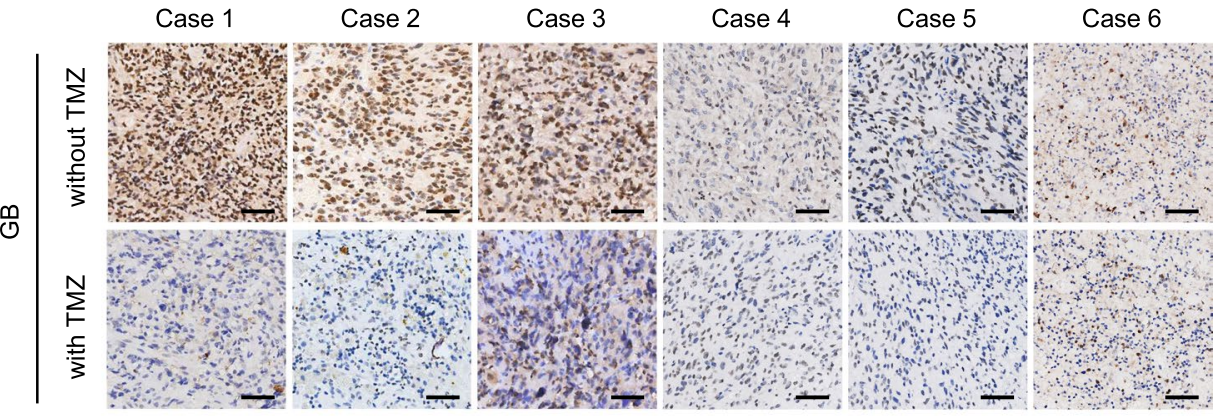

D

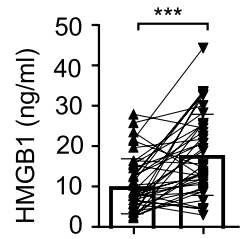

F

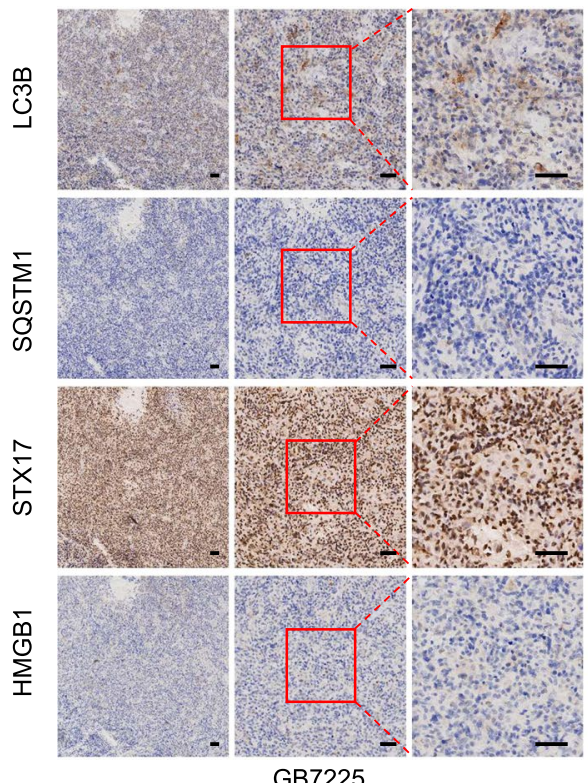

GB7225

E

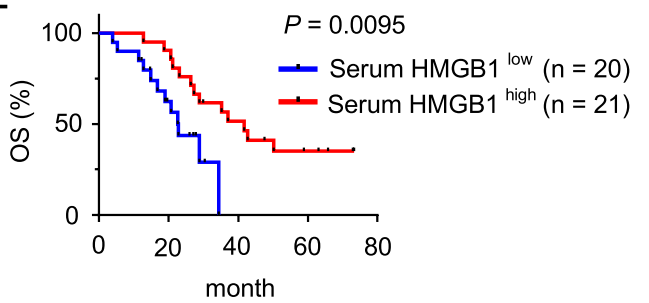

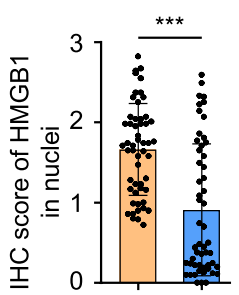

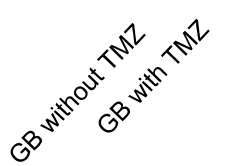

G
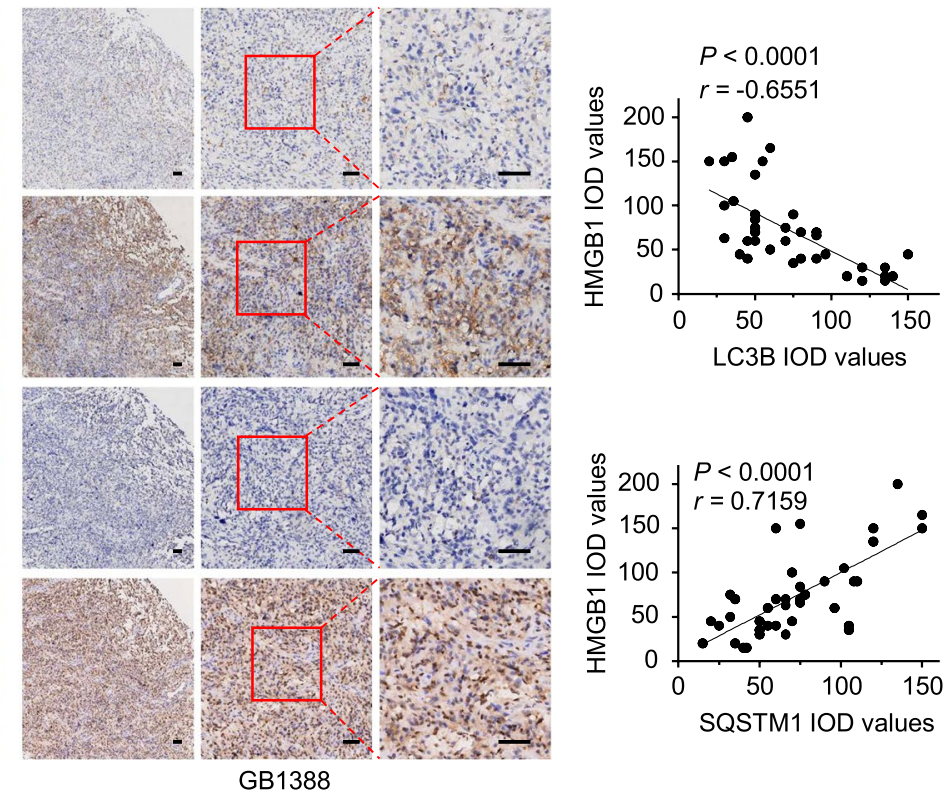

Fig. 2 (See legend on previous page.) 
without TMZ $(500 \mu \mathrm{M})$ for $48 \mathrm{~h}$ significantly decreased M1-like activation associated genes, CCL2, IL-1,$I L-6$ and TNF- $\alpha$ (Fig. 3E). Then, we knocked down HMGB1 with shRNA in GL261 cells and generate orthotopic intracranial model in C57/BL6 mice to confirm the effect of HMGB1 on M1-like TAM polarization. Flow cytometry was used to explore TAMs isolated from the intracranial xenograft tumors under TMZ treatment. The results showed that the percentage of $\mathrm{CD}^{+} 6^{+}$cells (M1-like marker) elevated, but the percentage of $\mathrm{CD}^{2} 6^{+}$in $\mathrm{CD} 45^{+}$ $\mathrm{CD}_{11 b^{+}}$cells from xenograft tumors of GL261 cells with Hmgb1 knockdown significantly reduced (Fig. 3F). Thus, GB-secreted HMGB1 upon TMZ treatment exerts a critical role in M1-like polarization of TAM.

\section{M1-like polarization of macrophages induced by HMGB1 enhances the sensitivity of GB to TMZ therapy}

Given the role of HMGB1 in the M1-like polarization of macrophages in vitro, we hypothesized that it may play a critical role in GB microenvironment to enhance anti-tumor immune responses. To analyze the effect of HMGB1 on GB progression, we implanted GL261 cells with luciferase intracranially into $\mathrm{C} 57 \mathrm{BL} / 6$ mice, with administration of TMZ or rmHMGB1 or both. After eight days of tumor cell implantation, mice were treated with TMZ ( $5 \mathrm{mg} / \mathrm{kg}$ per day, i.p., for 5 consecutive days) together with or without rmHMGB1 $(50 \mu \mathrm{g} / \mathrm{kg}$ per day, i.v., for 5 consecutive days). Tumor growth was monitored by bioluminescence using an In Vivo Imaging System (IVIS). Bioluminescent imaging indicated that combined treatment with rmHMGB1 significantly enhanced the anti-tumor effect of TMZ shown by markedly inhibited growth of GL261 cell-derived xenografts, while rmHMGB1 treatment alone exerted little benefits on the growth of GL261 cell-derived xenografts (Fig. 4AB). Consequently, mice treated with TMZ and rmHMGB1 exhibited a significantly extended survival (Fig. 4C). In contrast, rmHMGB1 treatment alone had not effect on animal survival. Then, we used flow cytometry to analyze the proportion of M1-like $\left(\mathrm{CD} 45^{+} \mathrm{CD} 11 \mathrm{~b}^{+} \mathrm{CD} 11 \mathrm{c}^{+}\right)$ and $\mathrm{M} 2$-like $\left(\mathrm{CD} 45^{+} \mathrm{CD} 11 \mathrm{~b}^{+} \mathrm{CD} 206^{+}\right)$TAM isolated from GL261 cell-derived xenografts. The results showed that the proportion of $\mathrm{CD} 11 \mathrm{c}^{+}$cells in the xenografts treated with TMZ or TMZ plus rmHMGB1 significantly increased (Fig. 4D). Meanwhile, a distinct reduction of the percentage of $\mathrm{CD}^{206^{+}}$cells was observed (Fig. 4E). Moreover, to determine extracellular HMGB1 distribution and its correlation with the subtype of TAMs in GB xenografts, frozen sections were stained for HMGB1 together with pan TAM marker IBA1 and the M1/M2-like TAM marker (M1: CD16/32; M2: CD206) in GL261 cellderived xenografts. We found that in HMGB1 ${ }^{\text {high }}$ region of GL261 cell-derived xenografts, M1-like TAMs markedly increased with significantly reduced M2-like TAMs (Fig. 4F-G and Fig. S7A-B). These data demonstrate that HMGB1 secreted by GB cells enhanced the sensitivity of the tumors to TMZ therapy by inducing M1-like polarization of macrophages. In addition, immunofluorescent staining showed that administration of TMZ plus rmHMGB1 reduced Ki67-postive proliferative cells and increased the number of apoptotic cells as marked by cleaved-caspase-3 in GL261 cell-derived xenografts (Fig. S8A-B). We further found that GB sections with high extracellular HMGB1 showed enhanced cleavedcaspase-3 staining in patients (GB0436 and GB8421) with TMZ treatment (Fig. S8C). These data demonstrate that HMGB1 secreted by GB upon TMZ treatment is a critical paracrine factor to mediate the tumor-suppressive effect of M1-like TAMs to restrain GB growth.

\section{RAGE is a receptor for HMGB1 on TAMs}

To explore the molecular mechanisms underlying M1-like polarization of macrophages promoted by HMGB1, we used co-immunoprecipitation (Co-IP) to examine the receptor interacting with HMGB1 expressed by macrophages. We demonstrated that RAGE showed a high affinity for HMGB1 in macrophages (Fig. 5A-B). To further confirm the specificity of RAGE as a receptor for HMGB1 expressed by macrophages, we used RFP or FITC labeled-rhHMGB1/rmHMGB1 to test its

\footnotetext{
(See figure on next page.)

Fig. 3 HMGB1 contributes to M1-like polarization of macrophages. A The mRNA expression of HMGB1 receptors (TLR2, TLR4, TLR9 and RAGE) in humanGB specimens from GEO database (Bone marrow derived macrophage-tumor associated macrophages, BMDM-TAMs; Macroglia-TAMs; Neoplastic cells; Oligodendrocyte progenitor cells, OPCs; Neural progenitor cells, NPCs; Endothelial cells, ECs; Pericytes, PCs; Peripheral blood lymphocytes, PBLs; Neurons.) was analyzed by single cell sequencing. B Immunofluorescence (IF) of HMGB1 receptors TLR2, TLR4, TLR9 and RAGE (green) in TAMs marked by IBA1 (red) in human GB samples (GB6429) without TMZ. Scale bars $=10 \mu \mathrm{m}$. C The mRNA level of M1-like phenotype genes in THP1 cell line-derived and RAW264.7 macrophages stimulated with $1 \mu \mathrm{g} / \mathrm{ml}$ recombinant human HMGB1 (rhHMGB1) or recombinant mouse HMGB1 (rmHMGB1) for $24 \mathrm{~h}$. D The mRNA level of M2-like phenotype genes in THP1 cell line-derived and RAW264.7 macrophages stimulated with $1 \mathrm{\mu g} / \mathrm{ml}$ rhHMGB1 or rmHMGB1 for $24 \mathrm{~h}$. E A scheme for in vitro co-culture system (left panel). THP1 cell line-derived macrophages (upper chamber) and shHMGB1 or shCtrl-LN229 cells (low chamber) were co-cultured for $24 \mathrm{~h}$ then with TMZ treatment for $24 \mathrm{~h}$. The expression of M1-like phenotype genes, CCL2, IL-1 1 , LL-6 and TNF- $\alpha$, was detected by qPCR (right panel). $\mathbf{F}$ Representative flow cytometric analysis of CD86 ${ }^{+}$

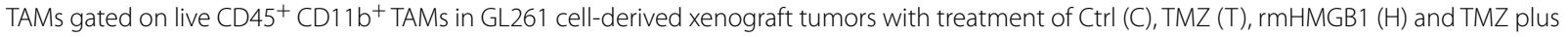
rmHMGB1 $(T+H)$ for 21 days (left panel). The histogram showed statistical analysis (right panel). $n=4 .{ }^{*} P<0.05,{ }^{* *} P<0.01,{ }^{* * *} P<0.001, n s=$ no significance
} 
A
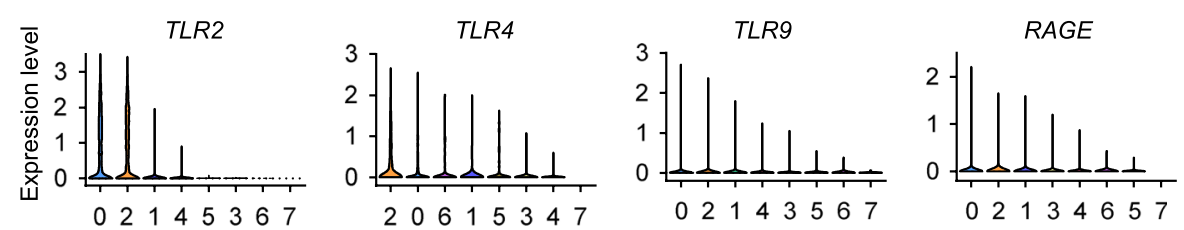

0. BMDM-TAMs, 2. Microglia-TAMs, 1.4. Neoplastic cells, 3. OPCs, 5. NPCs, 6. Vascular cells (ECs,PCs, PBLs), 7. Neurons.

B
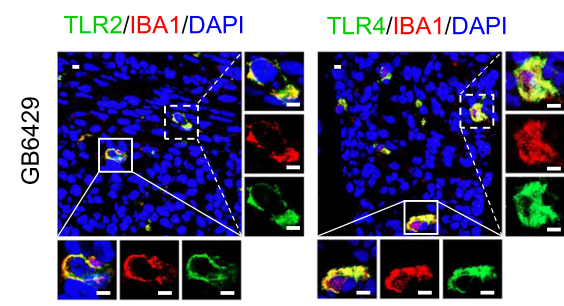

TLR9/IBA1/DAPI

RAGE/IBA1/DAPI

C

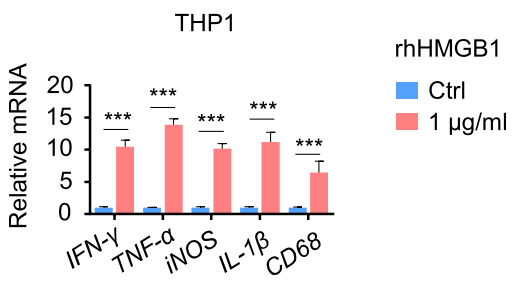

D

THP1

rhHMGB1

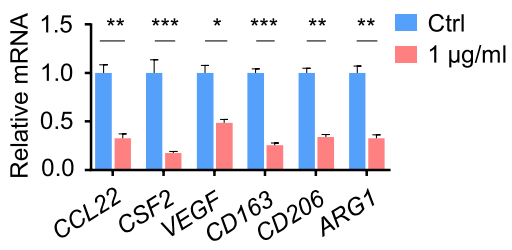

E

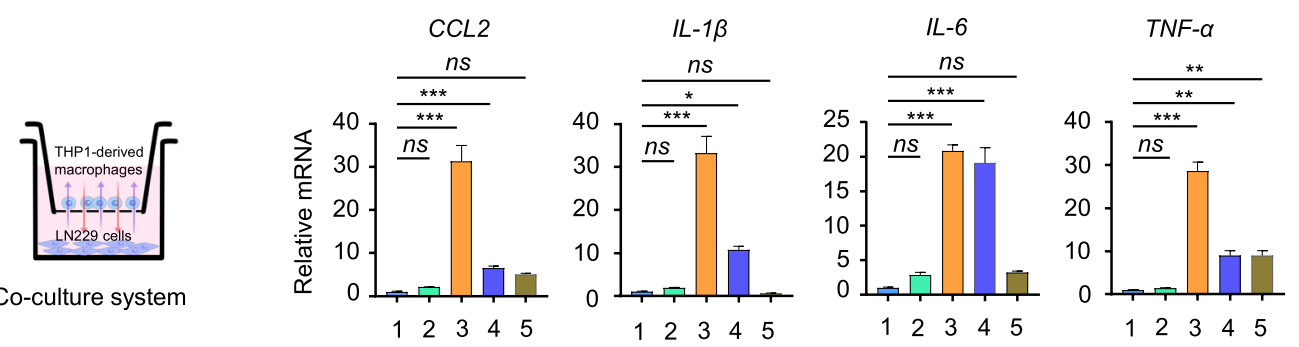

1: Ctrl; 2: LN229; 3: LN229 + TMZ; 4: shHMGB1-1 LN229 + TMZ; 5: shHMGB1-2 LN229 + TMZ

$\mathbf{F}$
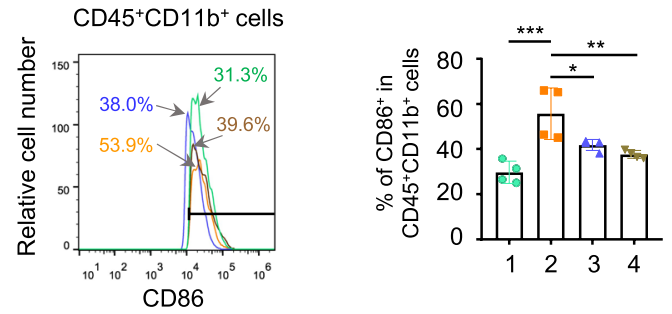

1: Ctrl 2: GL261+TMZ

3: shHmgb1-1 GL261 + TMZ

4: shHmgb1-2 GL261 + TMZ

Fig. 3 (See legend on previous page.)
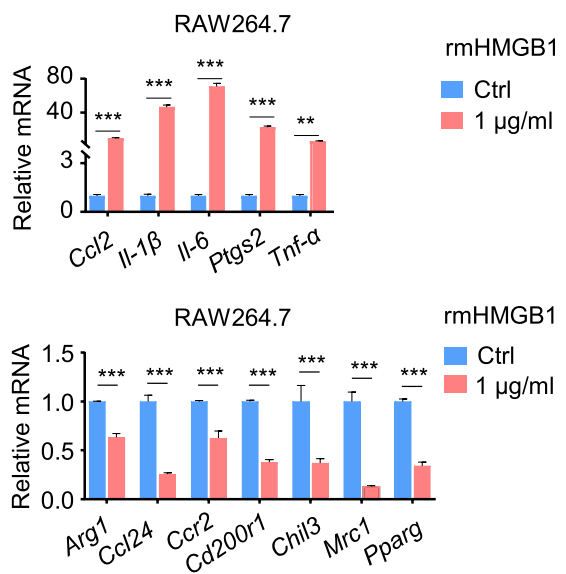
binding to macrophages. We found that the binding of rHMGB1 with RAGE on macrophages increased with the exposure time (Fig. 5C). Moreover, immunofluorescence showed co-expression of HMGB1 and RAGE on human GB samples (GB8060 and GB9080) with TMZ treatment (Fig. 5D). We also observed that the mRNA and protein levels of RAGE markedly increased with no change in the levels of other putative receptors on THP1 cell linederived macrophages and RAW264.7 macrophages after stimulation with rhHMGB1 or rmHMGB1 (Fig. 5E-F). These data indicate that RAGE is a major receptor on macrophages serving paracrine signaling in response to HMGB1 produced by GB cells upon TMZ treatment.

The RAGE-NFKB-NLRP3 inflammasome pathway is involved in M1-like polarization of macrophages via HMGB1

HMGB1 polarizes a M1-like phenotype of macrophages by promoting the release of pro-inflammatory cytokines through activation of inflammasomes [42, 43]. We found significantly increased levels of TNF- $\alpha$, IFN- $\gamma$, IL- $1 \beta$, IL-6, IL- 8 and CCL2 in the supernatants released by THP1 cell line-derived macrophages cultured with rhHMGB1. The effect of rhHMGB1 was reduced by addition of FPS-ZM1, an inhibitor of RAGE (Fig. 6A). After culture with CY-09, an inhibitor of NLRP3 which is a key component of the protein complex in inflammasome, the levels of pro-inflammatory cytokines produced by THP1 cell line-derived macrophages upon HMGB1 also significantly decreased (Fig. 6A). These results suggest that HMGB1 promotes the release of pro-inflammatory cytokines by macrophages through RAGE and inflammasome. In macrophages, HMGB1 activates RAGE through the phosphorylation of the extracellular regulated protein kinases (ERK1/2) followed by the activation of NFKB and cytokine production $[17,19,38]$. We found that rhHMGB1 increased the phosphorylation of ERK1/2, IKB and $\mathrm{NF} \kappa \mathrm{B}$ in THP1 cell line-derived macrophages (Fig. 6B), which was markedly suppressed by addition of FPS-ZM1 (Fig. 6C), indicating that RAGE activation by HMGB1 is linked to the phosphorylation of ERK1/2 and IKB that activates $\mathrm{NF \kappa B}$ to release pro-inflammatory cytokines by macrophages through NLRP3-dependent inflammasomes. To better exhibit the direct proof in vivo, we stained the sections from GL261 cell-derived xenografts treated with TMZ plus rmHMGB1, and found that the tumor regions with obvious extracellular HMGB1 highly expressed TNF- $\alpha$, IFN- $\gamma$, IL-1 $\beta$, IL-6, IL-8 and CCL2 detected by immunofluorescence, which are cytokines following the activation of RAGE-NFKB-NLRP3 inflammasome (Fig. 6D and Fig. S9A). Furthermore, our data showed that extracellular HMGB1 in xenografts induced the production of NLRP3 and ASC, the key component in inflammasome (Fig. S9B), which simultaneously promoted the release of TNF- $\alpha$, IFN- $\gamma$, IL- $1 \beta$, IL- 6 , IL- 8 and CCL2 in an NLRP3 inflammasome-dependent manner (Fig. S10). Collectively, the results confirmed the pathway of RAGE-NFkB-NLRP3 inflammasome via HMGB1 to induce M1-like polarization of TAMs in GB.

\section{Discussion}

Autophagy induction is one of the hallmark effects of anti-GB therapy with temozolomide (TMZ). Understanding the mechanisms of autophagy under TMZ treatment is crucial since chloroquine (CQ) and hydroxychloroquine (HCQ), which disrupt lysosomal acidification and manifest anti-cancer activity in mice, have not improved overall survival of GB patients when combined with TMZ [5].

Recent studies have demonstrated that autophagy not only controls protein degradation but also protein secretion known as secretory autophagy [8]. In this study, we demonstrated, for the first time, that extracellular HMGB1 released through secretory autophagy acts as a regulator of the crosstalk between GB cells and immune microenvironment that play major roles in tumor sensitivity towards TMZ. HMGB1 induces M1-like polarization of TAMs and promotes the secretion of proinflammatory cytokines, which trigger potent antitumor immunity [44] and converts an immunological "cold"

\footnotetext{
(See figure on next page.)

Fig. 4 HMGB1 enhances the sensitivity of TMZ therapy by inducing M1-like polarization of TAMs. A-B Bioluminescent images (A) and quantification of tumors in mice (B) implanted with GL261 cells with treatment of Ctrl, TMZ, rmHMGB1 and TMZ plus rmHMGB1 for 7, 14 and 21 days. C The survival curves of tumor-bearing mice implanted with GL261 cells for indicated treatments. $n=8$. D Representative flow cytometric analysis of tumor-infiltrating CD11 $\mathrm{C}^{+}$TAMs gated on live CD45 ${ }^{+} \mathrm{CD} 11 \mathrm{~b}^{+}$TAMs isolated from GL261 cell-derived xenograft tumors with treatment of Ctrl (C), TMZ (T), rmHMGB1 (H) and TMZ plus rmHMGB1 $(T+H)$ for 21 days (left panel). The histogram showed the statistical analysis (right panel). $n=4$. E Representative flow cytometric analysis of tumor-infiltrating CD206 ${ }^{+}$TAMs gated on live CD45+ CD1 1 b ${ }^{+}$TAMs in GL261 cell-derived xenograft tumors with treatment of $\mathrm{Ctrl}(\mathrm{C}), \mathrm{TMZ}(\mathrm{T})$, rmHMGB1 $(\mathrm{H})$ and TMZ plus rmHMGB1 $(\mathrm{T}+\mathrm{H})$ for 21 days (left panel). The histogram showed the statistical analysis (right panel). $n=4$. $\mathbf{F}$ Representative IF of HMGB1 (purple), pan macrophage marker IBA1 (red) and M1-like TAM marker CD16/32 (green) in GL261 cell-derived xenograft tumors treated with TMZ plus rmHMGB1 (left panel). Quantitation of IBA1 ${ }^{+} / \mathrm{CD} 16 / 32^{+}$TAM population in Ctrl, TMZ, rmHMGB1 and TMZ plus rmHMGB1 treatment groups (right panel). Scale bars $=50 \mu \mathrm{m}$. G Representative IF of HMGB1 (purple), IBA1 (red) and M2-like TAM marker CD206 (green) in GL261 cell-derived xenograft tumors treated with TMZ plus rmHMGB1 (left panel). Quantitation of IBA ${ }^{+}$/ CD206 ${ }^{+}$TAM population in Ctrl, TMZ, rmHMGB1 and TMZ plus rmHMGB1 treatment groups (right panel). Scale bars $=50 \mu \mathrm{m} .{ }^{*} P<0.05$, ${ }^{* *} P<0.01$, ${ }^{* * *} P<0.001, n s=$ no significance
} 
A

GL261-derived xenografts

Days 7 II

B

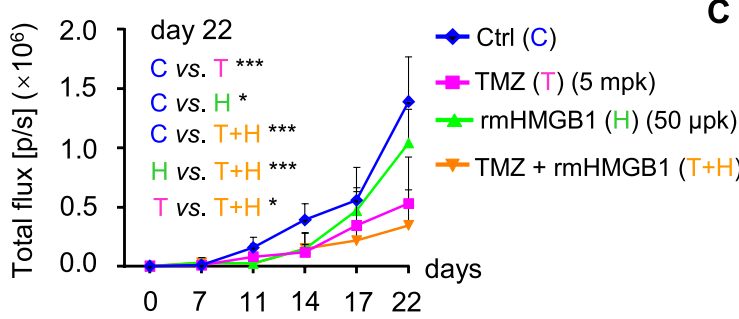

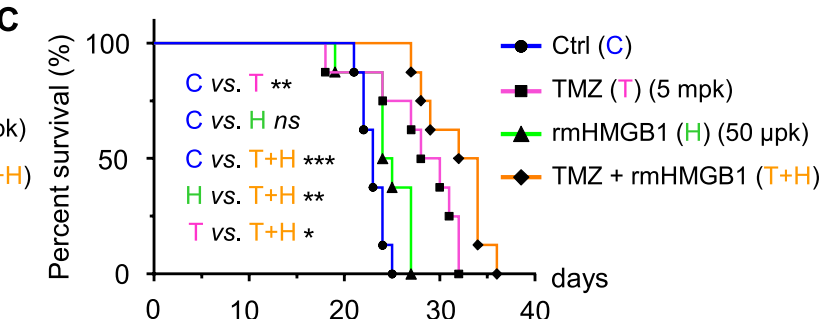

E

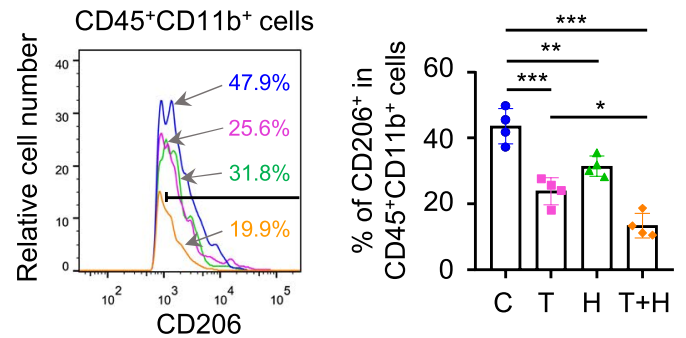

- TMZ + rmHMGB1 $(\mathrm{T}+\mathrm{H})$

F
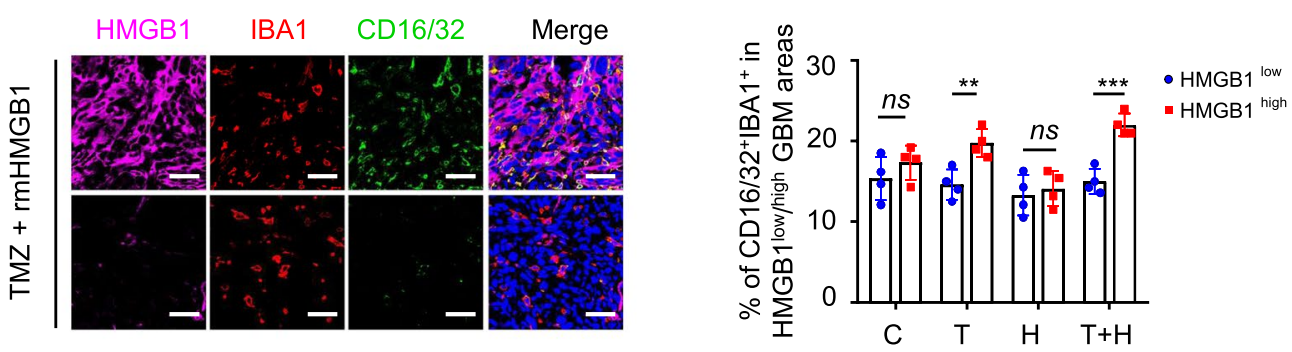

G
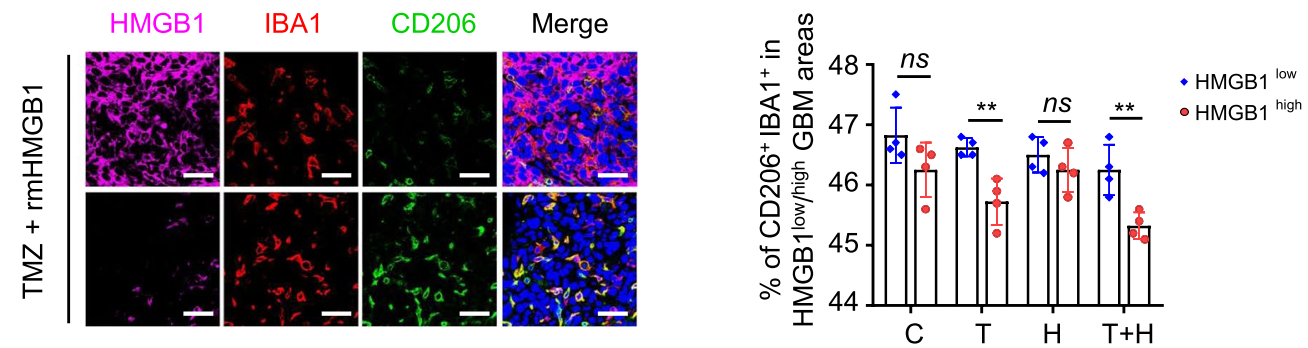

Fig. 4 (See legend on previous page.) 
microenvironment, which is frequently present in $\mathrm{GB}$, to an immunological "hot" one $[45,46]$.

Inhibition of autophagosome formation via LY294002 or 3-methyladenine (3-MA) in GB cells treated with TMZ decreased extracellular HMGB1, which highlighted the role of autophagy promoted by TMZ treatment. ATG5-mediated classical autophagy pathway and GORASP2, crucial for the unconventional secretion of cytoplasmic proteins $[8,47]$, have been reported to promote HMGB1 release by $293 \mathrm{~T}$ cells under starvation or lipopolysaccharide (LPS) treatment [8, 48]. Deficiency of ATG5 or GORASP2 in GB cells significantly reduced HMGB1 secretion during TMZ treatment, confirming that HMGB1 is contained in autophagosomes of GB cells.

Intracellular HMGB1 is stored in the nucleus as a DNA chaperone and loss of HMGB1 increases DNA damage and decreases DNA repair efficiency in response to chemotherapy [49]. Analysis of cancer genome databases revealed an inverse relationship between HMGB1 mRNA levels and overall survival of GB patients. Correlation between HMGB1 and TMZ treatment has been supported by measurement of HMGB1 expression levels via IHC and ELISA from paired GB patient samples. Under TMZ treatment, GB samples with lower HMGB1 IHC score in nuclei showed higher levels in sera. Higher HMGB1 levels in sera of GB patients favored overall survival indicating the increased sensitivity to TMZ. Culture of GB cells with rhHMGB1 in vitro did not show significant effect on the proliferation of GB cells (Fig. S11A). We also cultured GB cells with rhHMGB1 and TMZ in vitro, with no effect on the sensitivity of tumor cells to TMZ (Fig. S11B). Thus, extracellular HMGB1 secreted by GB cells under TMZ treatment have little effect on tumor cells.

Accumulating evidence shows that secreted proteins are responsible for the crosstalk among cells in the TME, which may play a critical role in the regulation of drug responses [50]. A recent study showed that HMGB1 acted as a ligand for toll-like receptor 2 (TLR2) on bone marrow-derived GB-infiltrating dendritic cells to elicit tumor regression [22]. TME of GB is composed of multiple components, including parenchymal cells, soluble factors, blood vessels, extracellular matrix and infiltrating immune cells in which TAMs constitute a dominant cell population $[51,52]$. Our study revealed that RAGE, the receptor for HMGB1, is mainly located in TAMs and is responsible for the crosstalk with HMGB1 in the GB microenvironment to regulate TMZ responses. It is well recognized that TAMs include two major populations: tumor-supportive M2-like macrophages and tumor-suppressive M1-like macrophages. In our study, we revealed that HMGB1 induced M1-like polarization of TAMs in TME to activate ERK1/2-NFKB-NLRP3 inflammasome pathway resulting in the release of cytokines IFN- $\gamma$, IL-1 $\beta$, IL-6, CCL2, TNF- $\alpha$ and IL-8. Knockdown of HMGB1 in GB cells abolished M1-like polarizations of TAMs in co-culture system upon TMZ treatment. HMGB1 in vivo significantly increased polarization of tumor-suppressive TAMs (M1-like) and inhibited intracranial GB growth. Therefore, both GB-secreted HMGB1 via secretory autophagy and TAMs constitute a paracrine signaling loop in TME which is critical for systemic antitumor immune responses.

It was previously reported that the polarization of M1-like macrophages is characterized by NFKB-NLRP3 activation $[53,54]$ and release of proinflammatory mediators including IFN- $\gamma$, IL- $1 \beta$, IL- 6 , CCL2, TNF- $\alpha$, and IL-8 $[43,55]$. Our study assessed ERK1/2, IKB and NFKBNLRP3 pathway and found that these molecules were activated in macrophages by HMGB1. Proinflammatory cytokines are elevated after stimulation of macrophages by rHMGB1 which signals through RAGE [56].

In summary, we demonstrated that enhanced secretory autophagy in GB under TMZ treatment facilitated M1-like polarization of TAMs to inhibit GB growth. HMGB1 acted as a key regulator in mediating the crosstalk between GB cells and tumor-suppressive M1-like TAMs in the TME with the presence of the chemotherapeutic agent TMZ (Fig. 7). This may explain at least in part the reason why autophagy inhibition in combination with TMZ failed to cause effective tumor regression. Our study suggests a novel approach to enhancing the chemosensitivity of GB by targeting the metabolic pathway of HMGB1 in TME.

\footnotetext{
(See figure on next page.)

Fig. 5 HMGB1 binds to RAGE on TAMs. A Co-IP assays of interaction of HMGB1 with RAGE in THP1 cell line-derived macrophages (left panel) and RAW264.7 macrophages (right panel). Cell lysates were immunoprecipitated with anti-HMGB1 antibody, then immunoblotted with anti-HMGB1 and anti-RAGE antibodies. B Co-IP assays of interaction of RAGE with HMGB1 in THP1 cell line-derived macrophages (left panel) and RAW264.7 macrophages (right panel). Cell lysates were immunoprecipitated with anti-RAGE antibody, then immunoblotted with anti-HMGB1 and anti-RAGE antibodies. C THP1 cell line-derived macrophages (left panel) and RAW264.7 macrophages (right panel) were treated with RFP-labeled rhHMGB1 or FITC-labeled rmHMGB1 for 5 and 30 min, respectively. Scale bars $=10 \mu \mathrm{m}$. D Colocalization of HMGB1 (green) and RAGE (red) in human GB samples (GB8060 and GB9080) with TMZ treatment. Scale bars = $10 \mu \mathrm{m}$. E The mRNA expression of HMGB1 receptors (TLR2, TLR4, TLR9, RAGE) by THP1 cell line-derived macrophages after stimulation with rhHMGB1 at indicated concentrations for $24 \mathrm{~h}$ (left panel). Immunoblot of RAGE in THP1 cell line-derived macrophages with or without rhHMGB1 treatment for $48 \mathrm{~h}$ (right panel). F The mRNA expression of HMGB1 receptors (T/r2, T/r4, TIr9, Rage) by RAW264.7 macrophages after stimulation with rmHMGB1 at indicated concentrations for $24 \mathrm{~h}$ (left panel). Immunoblot of RAGE in RAW264.7 macrophages with or without rmHMGB1 treatment for $48 \mathrm{~h}$ (right panel). ${ }^{*} P<0.05,{ }^{*} P<0.01,{ }^{* * *} P<0.001, n s=$ no significance
} 


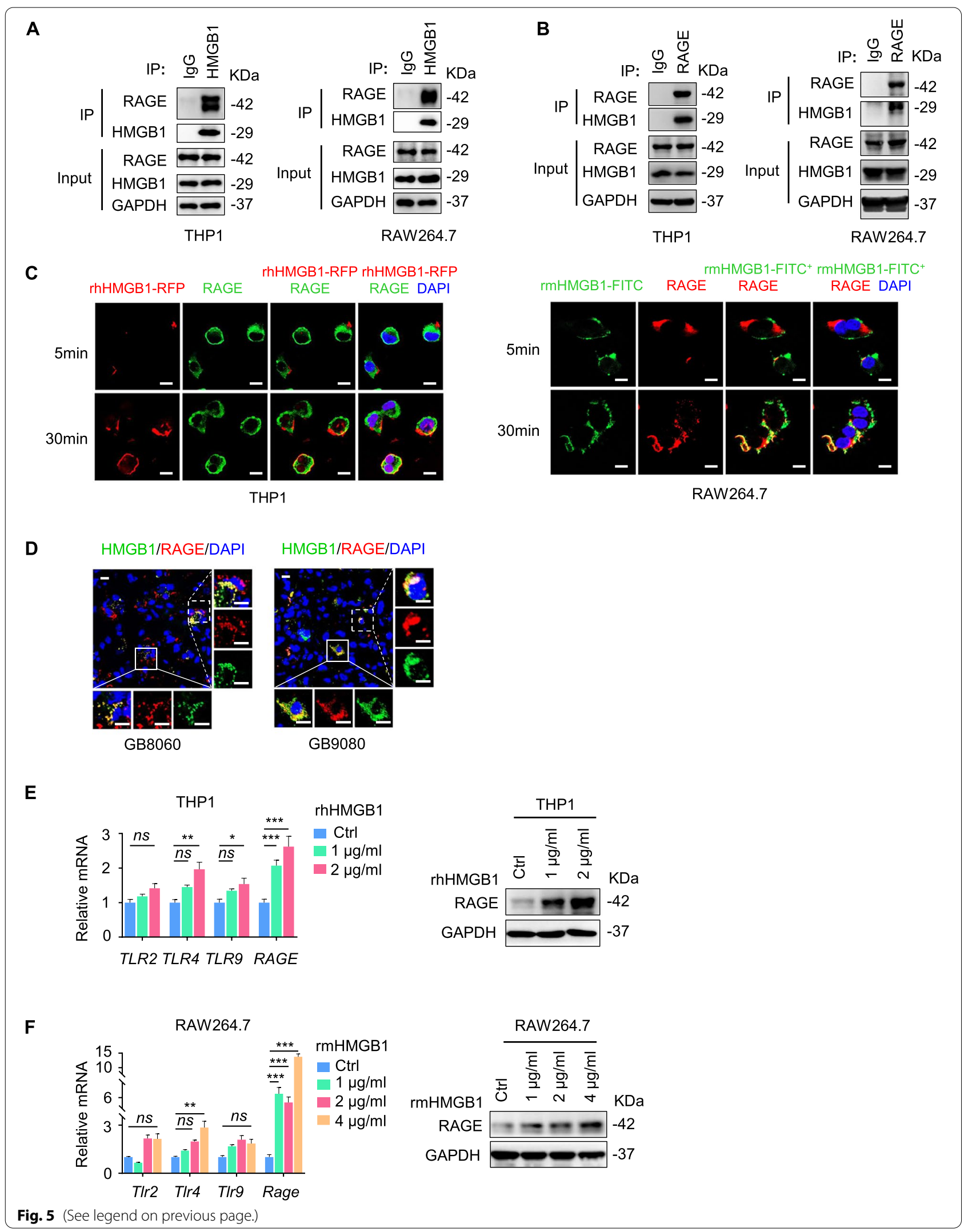




\section{A}
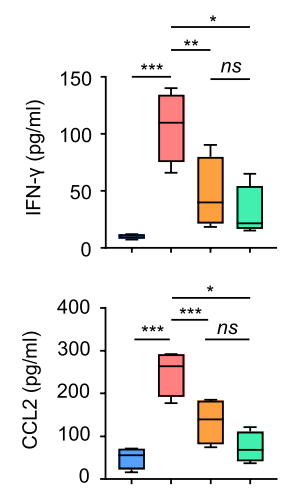

B

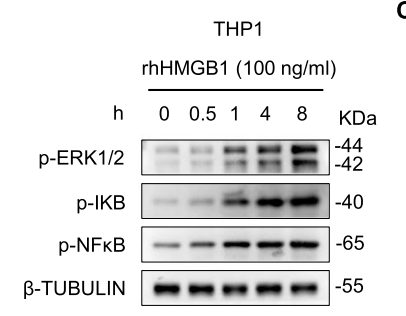

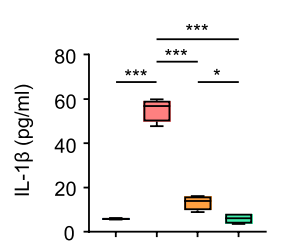
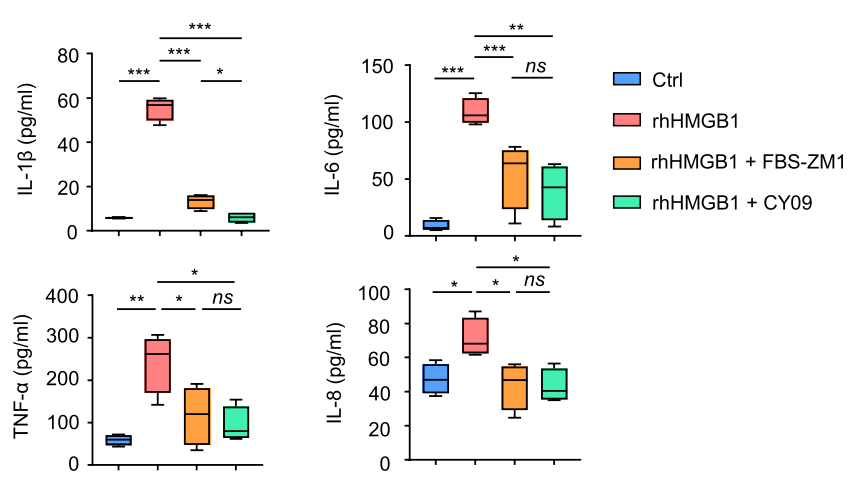

C

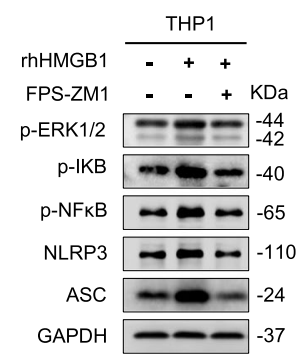

D

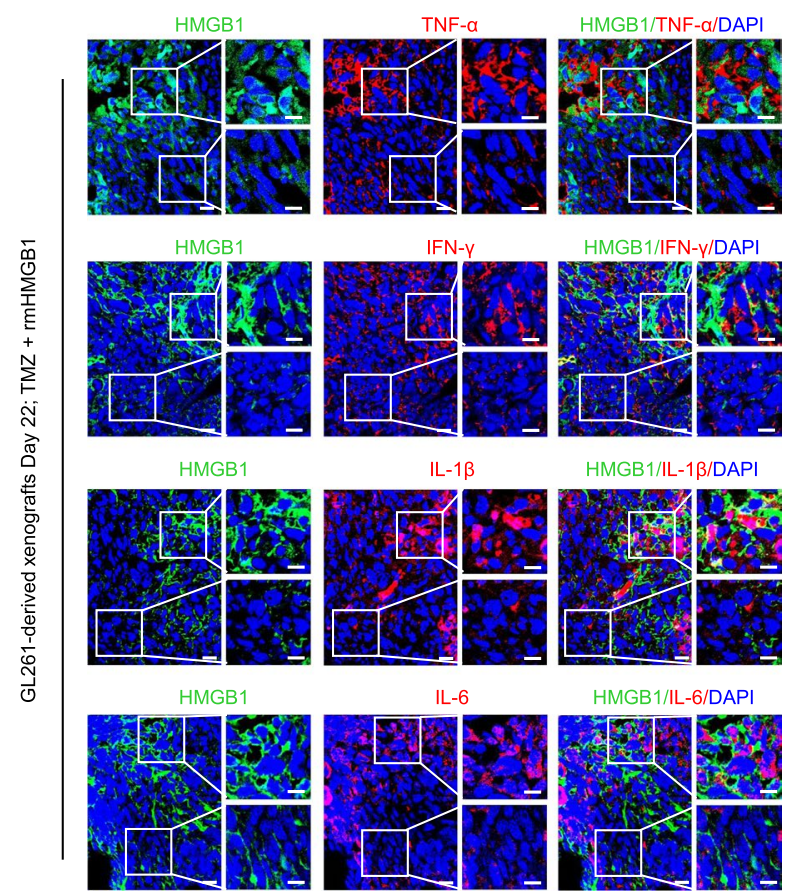

Fig. 6 HMGB1 enhances M1-like polarization of macrophages by activating ERK1/2-NFKB-NLRP3 inflammasome pathway. A Cytokines released

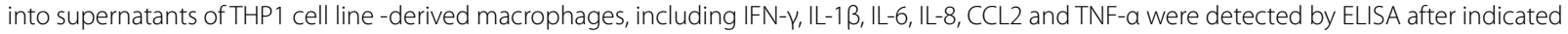
treatment for $24 \mathrm{~h}$. rhHMGB1: $1 \mu \mathrm{g} / \mathrm{ml}$; FBS-ZM1: $100 \mathrm{nM}$; CY-09: 5 MM. B Immunoblot of p-ERK1/2, p-IKB and p-NFKB in lysates of THP1 cell line-derived macrophages treated with rhHMGB1 $(100 \mathrm{ng} / \mathrm{ml})$ for indicated times. CTHP1 cell line-derived macrophages were pre-treated with FBS-ZM1 (100 nM) for $2 \mathrm{~h}$, then stimulated with rhHMGB1 $(1 \mathrm{\mu g} / \mathrm{ml})$ for $24 \mathrm{~h}$. p-ERK1/2, p-IKB, p-NFKB, NLRP3 and ASC levels were detected by immunoblot. D Representative IF of the cytokines (M1-like: TNF-a, IFN-y, IL-1 B, IL-6) (red) and HMGB1 (green) in GL261 cell-derived xenograft tumors treated with TMZ plus rmHMGB1. Squares are enlarged and shown on the right side of each image. Scale bars $=25 \mu \mathrm{m}$. ${ }^{*} P<0.05,{ }^{* *} P<0.01$, ${ }^{* * *} P<0.001, n s=$ no significance 

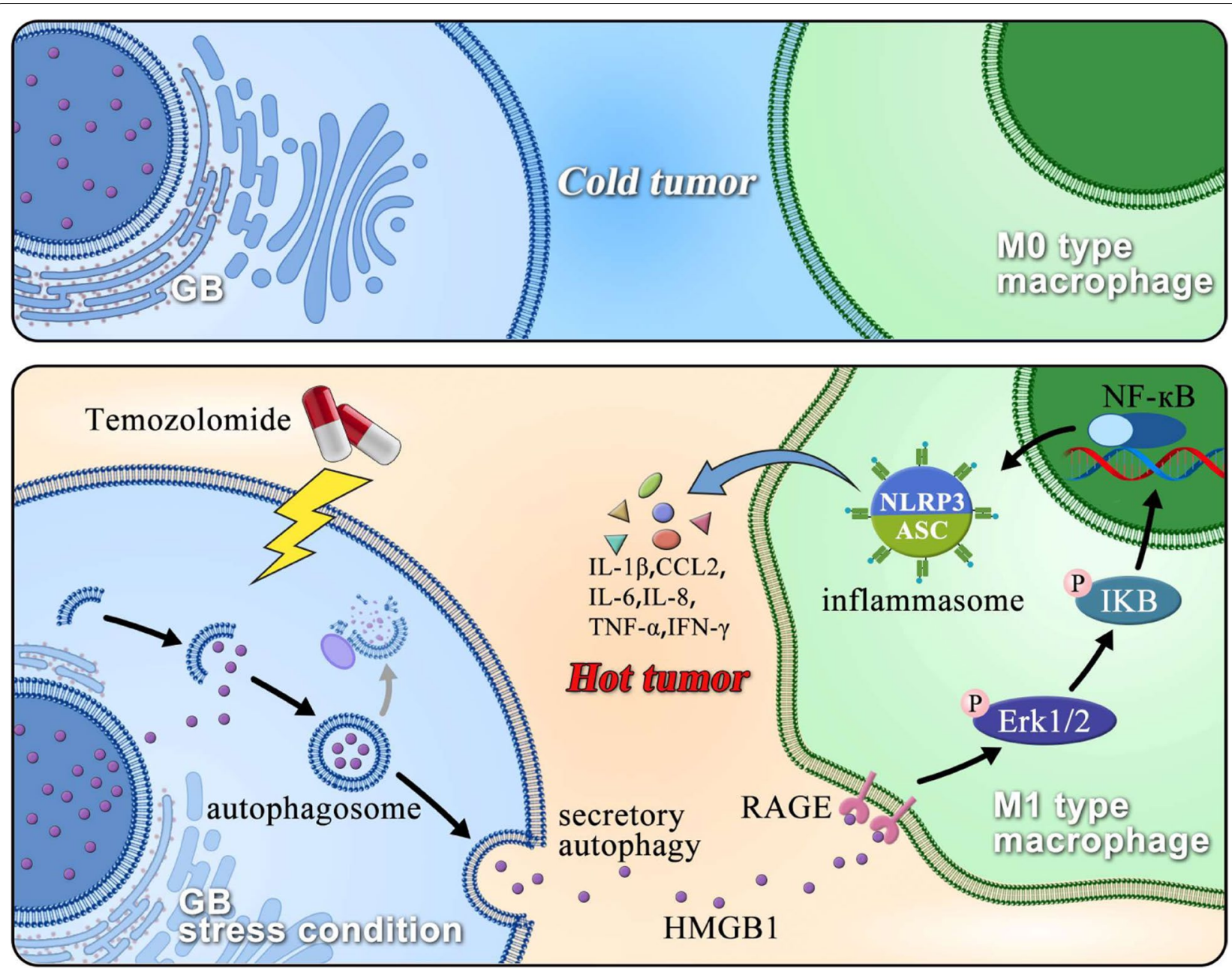

Fig. 7 A working model for the HMGB1 and GB chemosensitivity. When GBs are treated with TMZ chemotherapy, secretory autophagy is activated to release HMGB1 which promotes M1-like polarization of TAMs and TMZ chemosensitivity of GB

\section{Conclusions}

Our results indicate that enhanced secretory autophagy in GB with TMZ treatment is involved in the exocytosis of HMGB1. HMGB1 acts as a key regulator in the crosstalk between GB cells and tumor-suppressive M1-like TAMs in GB microenvironment and enhances TMZ sensitivity of GB cells. Thus, HMGB1 may be considered as an adjuvant for the chemotherapeutic agent TMZ.

\section{Abbreviations}

GB: Glioblastoma; GBM: Glioblastoma multiforme; TMZ: Temozolomide; HMGB1: High mobility group box 1;TEM: Transmission electron microscopy; ELISA: Enzyme-linked immunosorbent assay; qRT-PCR: Quantitative reverse transcription polymerase chain reaction; GFP: Green fluorescent protein; IHC: Immunohistochemistry; TME:Tumor microenvironment; TCGA: The cancer genome atlas; CGGA: The Chinese glioma genome atlas; LC3B: Microtubuleassociated protein 1 light chain; ATG5: Autophagy-related 5; ATG7: Autophagyrelated 7; BECN1: Beclin 1; SQSTM1: Sequestosome 1; STX17: Syntaxin 17;TLR2:
Toll-like receptor 2; TLR4:Toll-like receptor 4; TLR9: Toll-like receptor 9; RAGE: Receptor for advanced glycation end products; ERK1/2: Extracellular signalregulated protein kinases 1 and 2; IKB: Inhibitor of kappa-b; LPS: Lipopolysaccharide; DMSO: Dimethyl sulfoxide; NLRP3: NLR family pyrin domain containing 3; TAMs: Tumor-associated macrophages; CQ: Chloroquine; HCQ: Hydroxychloroquine; GORASP2: Golgi reassembly stacking protein 2.

\section{Supplementary Information}

The online version contains supplementary material available at https://doi. org/10.1186/s13046-022-02291-8.

Additional file 1: Figure S1. The levels of secretory autophagy proteins in the supernatants of $G B$ cells upon TMZ treatment.

Additional file 2: Figure S2. Involvement of secretory autophagy in the release of HMGB1 in GB upon TMZ treatment.

Additional file 3: Figure S3. Release of HMGB1 by TMZ-treated GB cells dependent on the formation of autophagic vacuoles.

Additional file 4: Figure S4. The correlation between mRNA HMGB1 and the prognosis of $\mathrm{GB}$ patients from database. 
Additional file 5: Figure S5. HMGB1 has no effect on microglia HMC3 Additional file 6: Figure S6. Knockdown of HMGB1 in LN229 cells and GL261 cells.

Additional file 7: Figure S7. The expression of M1/M2-like TAM markers in GL261 cell-derived xenograft tumors.

Additional file 8: Figure S8. The expression of apoptotic proteins in extracellular HMGB1-enriched regions of GL261 cell-derived xenograft tumors.

Additional file 9: Figure S9. The expression of cytokines released by the activation of RAGE-NFKB-NLRP3 inflammasome pathway in extracellular HMGB1-enriched regions of GL261 cell-derived xenograft tumors.

Additional file 10: Figure S10. The expression of cytokines released by the activation of RAGE-NFKB-NLRP3 inflammasome pathway in extracelIular NLRP3-enriched regions of GL261 cell-derived xenograft tumors.

Additional file 11: Figure S11. HMGB1 has no effect on GB cell proliferation in vitro.

Additional file 12: Table 1. Clinicopathological characteristics of human GBs used in this study. Table 2. Primers used for qRT-PCR. Table 3. The sequences of shRNA.

\section{Acknowledgements}

The authors thank Dr. Ji Ming Wang of Center for Cancer Research, National Cancer Institute at Frederick, for reviewing this article. The authors also acknowledge all the members of the Institute of Pathology and Southwest Cancer Center, Southwest Hospital, Third Military Medical University (Army Medical University).

\section{Authors' contributions}

X-HY, X-WB and ZL developed the hypothesis. ZL, W-JF, X-QC and SW performed the experiments, collected and analyzed the data. ML and S-SL partially performed the relevant experiments of intracranially orthotopic tumor model. K-DY, X-PT, R-SD, HZ, QN, YL, Q-RL,Y-FP, X-DL provided technical assistance or agents. ZL and X-HY wrote the manuscript. X-WB and $\mathrm{X}-\mathrm{HY}$ supervised the project. The author(s) read and approved the final manuscript.

\section{Funding}

This study was supported by grants from the National Natural Science Foundation of China (No. 81874087 and No. 31991172), Science and Technology Innovation Project of Chongqing Science and Technology Commission (No. cstc2018jcyj-yszXX0008) and the Graduate Scientific Research Innovation Project of Chongqing (No. CYS21514).

\section{Availability of data and materials}

All data used in this study are included within the article and additional files.

\section{Declarations}

\section{Ethics approval and consent to participate}

The use of human samples was approved by the Ethics Committee of Southwest Hospital, Third Military Medical University (Army Medical University) (KY2020294). All animal experiments were performed according to the guidelines for the care and use of laboratory animals and were approved by IACUC of Third Military Medical University (Army Medical University) (AMUWEC20201893).

\section{Consent for publication}

All authors have read and approved of its submission to this journal.

\section{Competing interests}

The authors declare no competing interests.

\section{Author details}

${ }^{1}$ Institute of Pathology and Southwest Cancer Center, Ministry of Education of China, Southwest Hospital, Third Military Medical University (Army Medical
University) and Key Laboratory of Tumor Immunopathology, Chongqing, China. ${ }^{2}$ Department of Nephrology, Southwest Hospital, Third Military Medical University (Army Medical University), Chongqing, China. ${ }^{3}$ Department of Oncology, Chinese Hainan Hospital of PLA General Hospital, Hainan Province, Sanya, China.

Received: 19 November 2021 Accepted: 16 February 2022

Published online: 22 February 2022

\section{References}

1. Stupp R, Mason WP, van den Bent MJ, Weller M, Fisher B, Taphoorn MJ et al. Radiotherapy plus concomitant and adjuvant temozolomide for glioblastoma. N Engl J Med. 2005;352(10):987-96. https://doi.org/10. 1056/NEJMoa043330

2. Legler JM, Ries LAG, Smith MA, Warren JL, Heineman EF, Kaplan RS, et al. Brain and Other Central Nervous System Cancers: Recent Trends in Incidence and Mortality. JNCI. 1999;91(16):1382-90. https://doi.org/10.1093/ jnci/91.16.1382

3. Arora A, Somasundaram K. Glioblastoma vs temozolomide: can the red queen race be won? Cancer Biol Ther. 2019;20(8):1083-90. https://doi. org/10.1080/15384047.2019.1599662.

4. Amaravadi RK, Kimmelman AC, Debnath J. Targeting Autophagy in Cancer: Recent Advances and Future Directions. Cancer Discov. 2019;9(9):1167-81. https://doi.org/10.1158/2159-8290.CD-19-0292.

5. Rosenfeld MR, Ye X, Supko JG, Desideri S, Grossman SA, Brem S, et al. A phase I/II trial of hydroxychloroquine in conjunction with radiation therapy and concurrent and adjuvant temozolomide in patients with newly diagnosed glioblastoma multiforme. Autophagy. 2014;10(8):135968. https://doi.org/10.4161/auto.28984.

6. Hombach-Klonisch S, Mehrpour M, Shojaei S, Harlos C, Pitz M, Hamai A, et al. Glioblastoma and chemoresistance to alkylating agents: Involvement of apoptosis, autophagy, and unfolded protein response. Pharmacol Ther. 2018;184:13-41. https://doi.org/10.1016/j.pharmthera. 2017.10.017.

7. Sotelo J, Briceño E, López-González MA. Adding chloroquine to conventional treatment for glioblastoma multiforme: a randomized, doubleblind, placebo-controlled trial. Ann Intern Med. 2006;144(5):337-43. https://doi.org/10.7326/0003-4819-144-5-200603070-00008.

8. Kim YH, Kwak MS, Lee B, Shin JM, Aum S, Park IH, et al. Secretory autophagy machinery and vesicular trafficking are involved in HMGB1 secretion. Autophagy. 2020:1-18. https://doi.org/10.1080/15548627.2020. 1826690.

9. Kimura T, Jia J, Claude-Taupin A, Kumar S, Choi SW, Gu Y, et al. Cellular and molecular mechanism for secretory autophagy. Autophagy. 2017;13(6):1084-5. https://doi.org/10.1080/15548627.2017.1307486.

10. New J, Arnold L, Ananth M, Alvi S, Thornton M, Werner L, et al. Secretory Autophagy in Cancer-Associated Fibroblasts Promotes Head and Neck Cancer Progression and Offers a Novel Therapeutic Target. Cancer Res. 2017;77(23):6679-91. https://doi.org/10.1158/0008-5472.CAN-17-1077.

11. Bel S, Pendse M, Wang Y, Li Y, Ruhn KA, Hassell B, et al. Paneth cells secrete lysozyme via secretory autophagy during bacterial infection of the intestine. Science. 2017;357(6355):1047-52. https://doi.org/10.1126/scien ce.aal4677.

12. Narita M, Young AR, Arakawa S, Samarajiwa SA, Nakashima T, Yoshida $S$, et al. Spatial coupling of mTOR and autophagy augments secretory phenotypes. Science. 2011;332(6032):966-70. https://doi.org/10.1126/ science.1205407.

13. Ponpuak M, Mandell MA, Kimura T, Chauhan S, Cleyrat C, Deretic V. Secretory autophagy. Curr Opin Cell Biol. 2015;35:106-16. https://doi.org/10. 1016/j.ceb.2015.04.016.

14. Davis S, Wang J, Ferro-Novick S. Crosstalk between the Secretory and Autophagy Pathways Regulates Autophagosome Formation. Dev Cell. 2017;41(1):23-32. https://doi.org/10.1016/j.devcel.2017.03.015.

15. Dupont N, Jiang S, Pilli M, Ornatowski W, Bhattacharya D, Deretic V. Autophagy-based unconventional secretory pathway for extracellular delivery of IL-1 beta. EMBO J. 2011;30(23):4701-11. https://doi.org/10. 1038/emboj.2011.398.

16. Zhao XL, Lin Y, Jiang J, Tang Z, Yang S, Lu L, et al. High-mobility group box 1 released by autophagic cancer-associated fibroblasts maintains 
the stemness of luminal breast cancer cells. J Pathol. 2017;243(3):376-89. https://doi.org/10.1002/path.4958.

17. Gaskell H, Ge X, Nieto N. High-Mobility Group Box-1 and Liver Disease. Hepatol Commun. 2018;2(9):1005-20. https://doi.org/10.1002/hep4.1223.

18. Lotze MT, Tracey KJ. High-mobility group box 1 protein (HMGB1): nuclear weapon in the immune arsenal. Nat Rev Immunol. 2005;5(4):331-42. https://doi.org/10.1038/nri1594.

19. Chiba S, Baghdadi M, Akiba H, Yoshiyama H, Kinoshita I, Dosaka-Akita $H$, et al. Tumor-infiltrating DCs suppress nucleic acid-mediated innate immune responses through interactions between the receptor TIM-3 and the alarmin HMGB1. Nat Immunol. 2012;13(9):832-42. https://doi.org/10. 1038/ni.2376.

20. Rivera Vargas T, Apetoh L. Danger signals: Chemotherapy enhancers? Immunol Rev. 2017;280(1):175-93. https://doi.org/10.1111/imr.12581.

21. Kang R, Zhang Q, Zeh HJ 3rd, Lotze MT, Tang D. HMGB1 in cancer: good, bad, or both? Clin Cancer Res. 2013;19(15):4046-57. https://doi.org/10. 1158/1078-0432.CCR-13-0495.

22. Curtin JF, Liu N, Candolfi M, Xiong W, Assi H, Yagiz K, et al. HMGB1 mediates endogenous TLR2 activation and brain tumor regression. PLoS Med. 2009;6(1): e10. https://doi.org/10.1371/journal.pmed.1000010.

23. Sica A, Mantovani A. Macrophage plasticity and polarization: in vivo veritas. J Clin Invest. 2012;122(3):787-95. https://doi.org/10.1172/JCI59643.

24. Murray PJ, Allen JE, Biswas SK, Fisher EA, Gilroy DW, Goerdt S, et al. Macrophage activation and polarization: nomenclature and experimental guidelines. Immunity. 2014;41(1):14-20. https://doi.org/10.1016/j.immuni. 2014.06.008.

25. Zheng W, Umitsu M, Jagan I, Tran CW, Ishiyama N, BeGora M, et al. An interaction between Scribble and the NADPH oxidase complex controls M1 macrophage polarization and function. Nat Cell Biol. 2016;18(11):1244-52. https://doi.org/10.1038/ncb3413.

26. Ubil E, Caskey L, Holtzhausen A, Hunter D, Story C, Earp HS. Tumorsecreted Pros 1 inhibits macrophage M1 polarization to reduce antitumor immune response. J Clin Investig. 2018;128(6):2356-69. https://doi.org/ 10.1172/jci97354.

27. Pinet S, Bessette B, Vedrenne N, Lacroix A, Richard L, Jauberteau MO, et al. TrkB-containing exosomes promote the transfer of glioblastoma aggressiveness to YKL-40-inactivated glioblastoma cells. Oncotarget. 2016;7(31):50349-64. https://doi.org/10.18632/oncotarget.10387.

28. Wang Z, Wang B, Shi Y, Xu C, Xiao HL, Ma LN, et al. Oncogenic miR-20a and miR-106a enhance the invasiveness of human glioma stem cells by directly targeting TIMP-2. Oncogene. 2015;34(11):1407-19. https://doi. org/10.1038/onc.2014.75.

29. Cao M, Cai J, Yuan Y, Shi Y, Wu H, Liu Q, et al. A four-gene signaturederived risk score for glioblastoma: prospects for prognostic and response predictive analyses. Cancer Biol Med. 2019;16(3):595-605. https://doi.org/10.20892/j.issn.2095-3941.2018.0277.

30. Shi Y, Chen C, Zhang X, Liu Q, Xu JL, Zhang HR, et al. Primate-specific miR663 functions as a tumor suppressor by targeting PIK3CD and predicts the prognosis of human glioblastoma. Clin Cancer Res. 2014;20(7):180313. https://doi.org/10.1158/1078-0432.CCR-13-2284.

31. Wu HB, Yang S, Weng HY, Chen Q, Zhao XL, Fu WJ, et al. Autophagyinduced KDR/NEGFR-2 activation promotes the formation of vasculogenic mimicry by glioma stem cells. Autophagy. 2017;13(9):1528-42. https://doi.org/10.1080/15548627.2017.1336277.

32. Klionsky DJ, Abdel-Aziz AK, Abdelfatah S, Abdellatif M, Abdoli A, Abel S, et al. Guidelines for the use and interpretation of assays for monitoring autophagy (4th edition)1. Autophagy. 2021;17(1):1-382. https://doi.org/ 10.1080/15548627.2020.1797280.

33. Mizushima N, Yoshimori T, Levine B. Methods in mammalian autophagy research. Cell. 2010;140(3):313-26. https://doi.org/10.1016/j.cell.2010.01. 028.

34. Zhang X, Wang Y. The Golgi stacking protein GORASP2/GRASP55 serves as an energy sensor to promote autophagosome maturation under glucose starvation. Autophagy. 2018;14(9):1649-51. https://doi.org/10. 1080/15548627.2018.1491214.

35 Zhang X, Wang L, Lak B, Li J, Jokitalo E, Wang Y. GRASP55 Senses Glucose Deprivation through O-GlcNAcylation to Promote AutophagosomeLysosome Fusion. Dev Cell. 2018;45(2):245-61. https://doi.org/10.1016/j. devcel.2018.03.023 (e6).

36. Yan W, Chang Y, Liang X, Cardinal JS, Huang H, Thorne SH, et al. Highmobility group box 1 activates caspase-1 and promotes hepatocellular carcinoma invasiveness and metastases. Hepatology. 2012;55(6):1863-75. https://doi.org/10.1002/hep.25572.

37. Liu Y, Yan W, Tohme S, Chen M, Fu Y, Tian D, et al. Hypoxia induced HMGB1 and mitochondrial DNA interactions mediate tumor growth in hepatocellular carcinoma through Toll-like receptor 9. J Hepatol. 2015;63(1):11421. https://doi.org/10.1016/j.jhep.2015.02.009.

38. Angelopoulou E, Piperi C, Adamopoulos C, Papavassiliou AG. Pivotal role of high-mobility group box 1 (HMGB1) signaling pathways in glioma development and progression. J Mol Med (Berl). 2016;94(8):867-74. https://doi.org/10.1007/s00109-016-1435-y.

39. Mukherjee A, Vasquez KM. Targeting Chromosomal Architectural HMGB Proteins Could Be the Next Frontier in Cancer Therapy. Cancer Res. 2020;80(11):2075-82. https://doi.org/10.1158/0008-5472.CAN-19-3066.

40. Deng M, Tang Y, Li W, Wang X, Zhang R, Zhang X, et al. The Endotoxin Delivery Protein HMGB1 Mediates Caspase-11-Dependent Lethality in Sepsis. Immunity. 2018;49(4):740-53. https://doi.org/10.1016/j.immuni. 2018.08.016 (e7).

41. Takenaka MC, Gabriely G, Rothhammer V, Mascanfroni ID, Wheeler MA, Chao CC, et al. Control of tumor-associated macrophages and T cells in glioblastoma via AHR and CD39. Nat Neurosci. 2019;22(5):729-40. https:// doi.org/10.1038/s41593-019-0370-y.

42. Schmieder A, Michel J, Schonhaar K, Goerdt S, Schledzewski K. Differentiation and gene expression profile of tumor-associated macrophages. Semin Cancer Biol. 2012;22(4):289-97. https://doi.org/10.1016/j.semca ncer.2012.02.002.

43. Wu K, Yuan Y, Yu H, Dai X, Wang S, Sun Z, et al. The gut microbial metabolite trimethylamine N-oxide aggravates GVHD by inducing M1 macrophage polarization in mice. Blood. 2020;136(4):501-15. https://doi. org/10.1182/blood.2019003990.

44. Grunhagen DJ, de Wilt JH, ten Hagen TL, Eggermont AM. Technology insight: Utility of TNF-alpha-based isolated limb perfusion to avoid amputation of irresectable tumors of the extremities. Nat Clin Pract Oncol. 2006;3(2):94-103. https://doi.org/10.1038/ncponc0426.

45. Weiss T, Puca E, Silginer M, Hemmerle T, Pazahr S, Bink A, et al. Immunocytokines are a promising immunotherapeutic approach against glioblastoma. Sci Transl Med. 2020;12(564). https://doi.org/10.1126/scitranslmed. abb2311.

46. Vom Berg J, Vrohlings M, Haller S, Haimovici A, Kulig P, Sledzinska A, et al. Intratumoral IL-12 combined with CTLA-4 blockade elicits T cell-mediated glioma rejection. J Exp Med. 2013;210(13):2803-11. https://doi.org/ 10.1084/jem.20130678.

47. Kinseth MA, Anjard C, Fuller D, Guizzunti G, Loomis WF, Malhotra V. The Golgi-associated protein GRASP is required for unconventional protein secretion during development. Cell. 2007;130(3):524-34. https://doi.org/ 10.1016/j.cell.2007.06.029.

48. Luo P, Xu Z, Li G, Yan H, Zhu Y, Zhu H, et al. HMGB1 represses the anticancer activity of sunitinib by governing TP53 autophagic degradation via its nucleus-to-cytoplasm transport. Autophagy. 2018;14(12):2155-70. https://doi.org/10.1080/15548627.2018.1501134.

49. Yuan S, Liu Z, Xu Z, Liu J, Zhang J. High mobility group box 1 (HMGB1): a pivotal regulator of hematopoietic malignancies. J Hematol Oncol. 2020;13(1):91. https://doi.org/10.1186/s13045-020-00920-3.

50. Zhang XN, Yang KD, Chen C, He ZC, Wang QH, Feng H, et al. Pericytes augment glioblastoma cell resistance to temozolomide through CCL5-CCR5 paracrine signaling. Cell Res. 2021. https://doi.org/10.1038/ s41422-021-00528-3.

51. Zhou W, Ke SQ, Huang Z, Flavahan W, Fang X, Paul J, et al. Periostin secreted by glioblastoma stem cells recruits $\mathrm{M} 2$ tumour-associated macrophages and promotes malignant growth. Nat Cell Biol. 2015;17(2):17082. https://doi.org/10.1038/ncb3090.

52. Lathia JD, Heddleston JM, Venere M, Rich JN. Deadly teamwork: neural cancer stem cells and the tumor microenvironment. Cell Stem Cell. 2011;8(5):482-5. https://doi.org/10.1016/j.stem.2011.04.013.

53. Jourdan T, Godlewski G, Cinar R, Bertola A, Szanda G, Liu J, et al. Activation of the Nlrp3 inflammasome in infiltrating macrophages by endocannabinoids mediates beta cell loss in type 2 diabetes. Nat Med. 2013;19(9):1132-40. https://doi.org/10.1038/nm.3265.

54. Lv LL, Feng $Y$, Wu M, Wang B, Li ZL, Zhong $X$, et al. Exosomal miRNA19b-3p of tubular epithelial cells promotes M1 macrophage activation in kidney injury. Cell Death Differ. 2020;27(1):210-26. https://doi.org/10. 1038/s41418-019-0349-y. 
55. Lu G, Zhang R, Geng S, Peng L, Jayaraman P, Chen C, et al. Myeloid cell-derived inducible nitric oxide synthase suppresses M1 macrophage polarization. Nature Communications. 2015;6(1). https://doi.org/10.1038/ ncomms7676.

56. Son M, Porat A, He M, Suurmond J, Santiago-Schwarz F, Andersson $\mathrm{U}$, et al. C1q and HMGB1 reciprocally regulate human macrophage polarization. Blood. 2016;128(18):2218-28. https://doi.org/10.1182/ blood-2016-05-719757.

\section{Publisher's Note}

Springer Nature remains neutral with regard to jurisdictional claims in published maps and institutional affiliations.

- fast, convenient online submission

- thorough peer review by experienced researchers in your field

- rapid publication on acceptance

- support for research data, including large and complex data types

- gold Open Access which fosters wider collaboration and increased citations

- maximum visibility for your research: over $100 \mathrm{M}$ website views per year

At BMC, research is always in progress.

Learn more biomedcentral.com/submissions 